\title{
A numerical study of the effects of orography on supercells
}

\author{
Paul M. Markowski ${ }^{\mathrm{a}, *}$, Nikolai Dotzek ${ }^{\mathrm{b}, \mathrm{c}, \dagger}$ \\ a Department of Meteorology, Pennsylvania State University, University Park, PA, USA \\ ${ }^{\mathrm{b}}$ Deutsches Zentrum für Luft- und Raumfahrt (DLR), Institut für Physik der Atmosphäre, Oberpfaffenhofen, Germany \\ c European Severe Storms Laboratory (ESSL), Münchner Str.20, Wessling, Germany
}

\section{A R T I C L E I N F O}

\section{Article history:}

Received 26 January 2010

Accepted 28 December 2010

\section{Keywords:}

Supercell

Thunderstorm

Mountains

Mesocyclone

\begin{abstract}
A B S T R A C T
The effects of idealized two- and three-dimensional terrain on a cyclonically rotating supercell thunderstorm are studied with a numerical model. The airflow over the terrain produces horizontal heterogeneity in the characteristics of the soundings and hodographs, which, in horizontally homogeneous environments, are the primarily factors that influence storm structure and evolution. Indeed, many of the differences between control simulations that feature storms over flat terrain and simulations in which terrain variations are introduced (e.g., a hill, escarpment, and valley) can be ascribed to differences in the storm environments, especially the thermodynamic conditions (variations in convective inhibition and relative humidity have the biggest effect on the simulated storms), caused by the airflow over and/or around the terrain. Regions of downsloping winds tend to be regions of enhanced convective inhibition and reduced relative humidity. Accordingly, there is a tendency for the simulated supercells to weaken (in terms of the intensities of their updrafts and mesocyclones) in the lee of terrain features where downsloping is present. Though most aspects of convective storm dynamics are independent of the ground-relative winds and only depend on the storm-relative winds, the ground-relative wind profile is of leading-order importance in determining the impact of the underlying terrain on the storms that cross it; the ground-relative wind profile dictates where winds will blow upslope or downslope, which controls to a large extent the manner in which the environment is modified.

When three-dimensional terrain is introduced (e.g., an isolated hill, a gap incised into a ridge), the resulting horizontal heterogeneity in the thermodynamic and vertical wind shear fields is considerably more complex than in the case of two-dimensional terrain (e.g., an infinitely long hill, valley, or escarpment). The effect of three-dimensional terrain on the storm environment can be further complicated by the generation of mesoscale vertical vorticity anomalies. In some cases, the interaction of supercells with preexisting lee vorticity anomalies can briefly enhance low-level rotation within the storm; however, the dominant role of three-dimensional terrain generally is its modification of soundings and hodographs, as is the case for two-dimensional terrain.
\end{abstract}

(c) 2011 Elsevier B.V. All rights reserved.

\section{Introduction}

Despite decades of observing and simulating deep moist convection, our understanding of how the underlying

\footnotetext{
* Corresponding author.

E-mail address: pmarkowski@psu.edu (P.M. Markowski).

$\dagger$ Deceased.
}

orography influences convective storms remains extremely limited. Little is known about the sensitivity of convective storms to the lower boundary condition, in general. Historically, most numerical simulations have used a flat, free-slip, non-conducting lower boundary. In this article we report on our recent investigation of the effects of idealized orography on supercell storms. Additional ongoing research is examining the sensitivities of convective storms to other aspects of 
the lower boundary, for example, the influence of cloud shading and associated modifications of the surface energy budget (Markowski and Harrington, 2005; Frame et al., 2008, 2009; Frame and Markowski, 2010), and the possible effects of environmental heterogeneity associated with a convective boundary layer driven by a surface heat flux (Knopfmeier et al., 2008; Nowotarski et al., 2010).

Although many investigators, at least anecdotally, express little doubt that terrain can have an appreciable effect on convective storms, there are few formal papers on the influence of terrain on convective storms. The primary difficulty with observational studies (e.g., Hannesen et al., 1998, 2000; LaPenta et al., 2005; Bosart et al., 2006) is that it is never possible to know how the storms would have evolved in the absence of terrain. Thus, observational work tends to remain fairly speculative about the impact of terrain on the observed structure and evolution of convection. A numerical modeling approach ought to be better suited for this line of work, for models allow the user to compare a simulation with terrain against a simulation without terrain (e.g., Frame and Markowski, 2006; Ćurić et al., 2007).

Frame and Markowski (2006) and Reeves and Lin (2007) previously have studied the effects of mountain ridges on mesoscale convective systems (MCSs). It was found that the forward speed and depth of the outflow are affected by its passage over a terrain barrier, with the outflow slowing and thinning as the mountain crest is approached, and then accelerating and deepening rapidly in the lee of the barrier, often forming a hydraulic jump. Because the evolution of an MCS is critically tied to the behavior of the cold pool-the MCS is maintained by the continuous triggering of new cells by the cold pool-terrain-induced modifications of cold pool evolution and structure unavoidably affect the evolution and structure of the MCS. Frame and Markowski (2006) found that many MCSs weaken as they approach a mountain crest and then reintensify in the lee of the mountain where a hydraulic jump develops in the outflow (i.e., where the outflow depth rapidly deepens).

Ćurić et al. (2007) simulated an isolated cumulonimbus cloud in an environment containing relatively strong vertical wind shear, with and without underlying terrain. The underlying terrain was that of the mountainous part of the Western Morava basin of Serbia. A number of differences were found between simulations with and without terrain, for example, storm-splitting occurred later and the counterrotating vortices were weaker in the simulation with terrain. No dynamical explanation was offered for how the terrain led to the differences in storm evolution and structure.

The present study on the influence of terrain on supercells uses idealized terrain rather than actual terrain. It is much easier for us to develop a dynamical understanding of the cause-and-effect relationship of the storm-terrain interactions if the terrain configuration is kept simple. In the next section, we elaborate on our methodology, and in Sections 3-5, we present the results. Control simulations (i.e., those with flat terrain) are described briefly (Section 3), followed by the results of simulations with two-dimensional terrain features (an isolated ridge, an escarpment, and a valley, all oriented in the north-south direction; Section 4) and three-dimensional terrain features (an isolated hill and channeled flow through a gap in a north-south-oriented ridge; Section 5). A summary and closing remarks appear in Section 6.

\section{Methodology}

The simulations were performed using the Bryan Cloud Model 1 (CM1 version 1, release 13) described by Bryan and Fritsch (2002) and Bryan (2002). The terrain-following coordinate of Gal-Chen and Somerville (1975) is used, and the governing equations are integrated using the RungeKutta technique described by Wicker and Skamarock (2002). The advection terms are discretized using fifth-order spatial discretization; no artificial diffusion is applied. The subgrid turbulence parameterization is similar to the parameterization of Deardorff (1980). The microphysics parameterization includes ice and is the NASA-Goddard version of the LinFarley-Orville (Lin et al., 1983) scheme.

The horizontal grid spacing is $500 \mathrm{~m}$; the vertical grid spacing varies from $100 \mathrm{~m}$ in the lowest $1 \mathrm{~km}$, to $500 \mathrm{~m}$ at the top of the domain. The domain is $100 \mathrm{~km} \times 250 \mathrm{~km} \times 18 \mathrm{~km}$ in the $x, y$, and $z$ directions, respectively. The grid is stationary, that is, the grid does not move with the storms (we were uncertain what unintended effects might arise with the introduction of terrain undulations if grid translation was employed). The large (small) time step is $3(0.3) \mathrm{s}$. Simulations were carried out for $4 \mathrm{~h}$.

The lower and upper boundaries are free-slip (the results reported herein were qualitatively unaffected when surface drag was imposed at the lower boundary). A Rayleigh damping layer (Durran and Klemp, 1983) occupies the uppermost $4 \mathrm{~km}$ of the model domain in order to damp gravity waves that propagate upward from the terrain and convection. An open-radiative boundary condition is applied along the lateral boundaries, where the speed of gravity wave propagation is estimated by vertically averaging outwarddirected gravity wave phase speeds along the lateral boundaries, with the inward-directed phase speed set to zero before averaging (Durran and Klemp, 1983).

There are no surface heat fluxes; although it is wellknown that circulations generated by the heating of sloping or elevated terrain are often important in the initiation of convective storms, the focus of this study is on the interaction of mature storms with terrain rather than the role of terrain in convection initiation. No atmospheric radiative heating is considered either, and there is no Coriolis force. The absence of surface heat fluxes, radiative forcing, and the Coriolis force allows the model environment to remain steady during the simulations, at least far from the influence of the terrain (the model fields unavoidably evolve in the vicinity of the terrain in the early stages of the simulations owing to the airflow over the terrain).

The environments of the simulated storms are initialized with a sounding very similar to that used by Weisman and Klemp (1982) (Fig. 1a). The analytic function used to define the vertical profile of relative humidity has the same form as that used by Weisman \& Klemp, but it has an exponent of 0.75 rather than 1.25. This results in our sounding being drier than the Weisman \& Klemp sounding in the layer that is immediately above the constant-mixing ratio layer in contact with the surface. In our initial experiments using the original Weisman \& Klemp sounding, the orographic ascent over even a small hill commonly resulted in the formation of a moist absolutely unstable layer (Bryan and Fritsch, 2000); we did not want the interpretation of our results to be complicated 


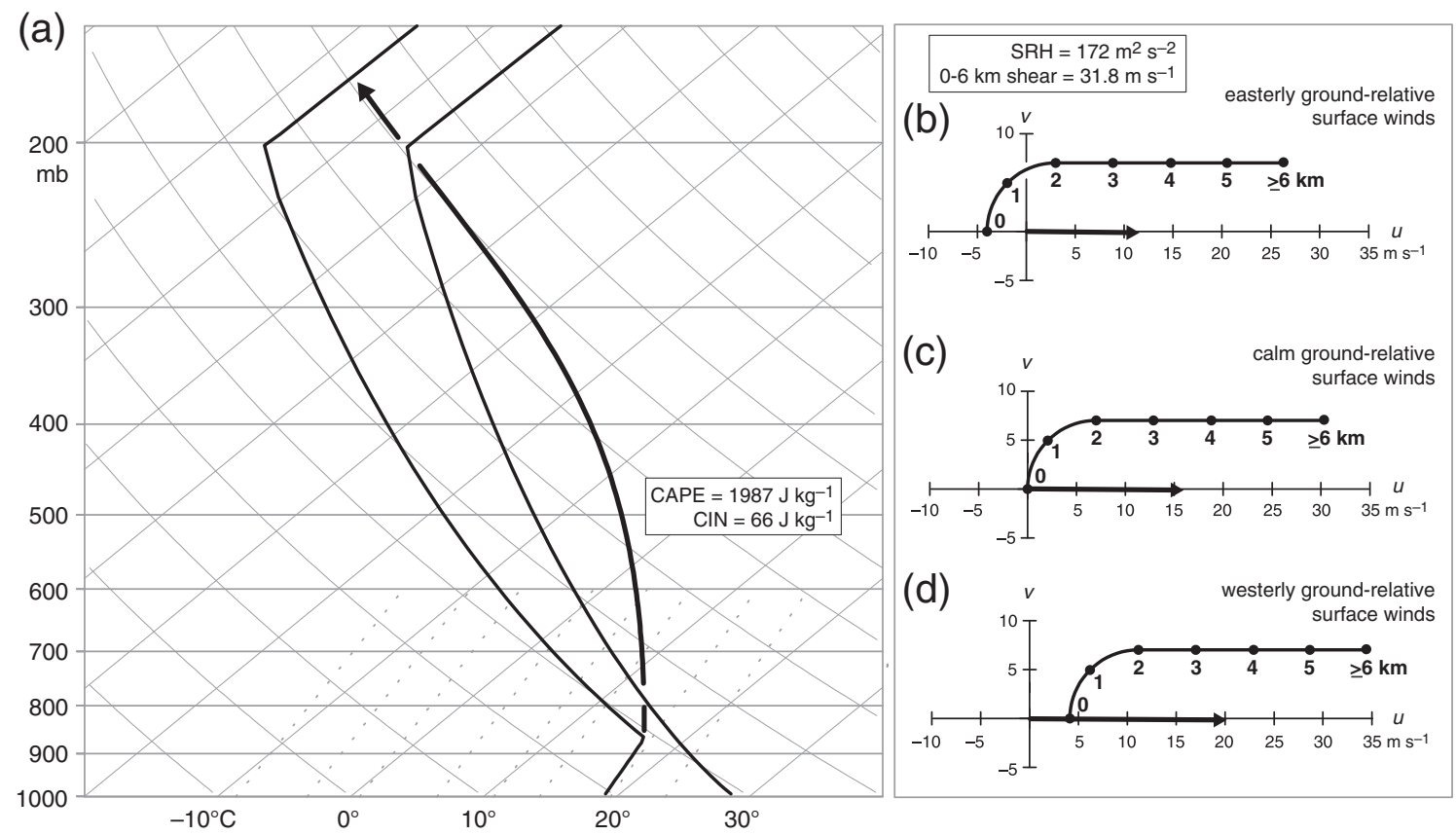

Fig. 1. (a) Skew $T$ - $\log p$ diagram of the sounding used to initialize the numerical simulations. The CAPE and CIN are also indicated for the parcel process curve shown (the CAPE and CIN calculations include the effects of moisture on buoyancy and are based on the pseudoadiabatic ascent of a parcel lifted from the surface). (b)-(d) Hodographs used to initialize the numerical simulations. The three hodographs have identical shapes and lengths, but have different profiles of groundrelative winds because of their different positions relative to the origin. The mean motions of the right-moving supercells are indicated by the bold vectors, and numerals along the hodograph traces indicate heights above ground level in kilometers. The SRH and 0-6 km shear vector magnitude also are indicated (they are the same for all three hodographs).

by dynamics associated with a moist absolutely unstable layer. The sounding has a surface-based convective available potential energy (CAPE) of $1987 \mathrm{~J} \mathrm{~kg}^{-1}$ and a surface-based convective inhibition (CIN) of $66 \mathrm{~J} \mathrm{~kg}^{-1}$. The CAPE and CIN calculations include the effects of moisture on buoyancy, neglect freezing, and are based on the pseudoadiabatic ascent of a parcel lifted from the surface. All CAPE and CIN values cited in this paper are computed in this manner.

The environmental wind profile is defined by the quartercircle hodograph used by Rotunno and Klemp (1982) (Fig. 1bd). The hodograph was shifted with respect to the origin in three different ways in order to vary the ground-relative winds. One wind profile has $4 \mathrm{~m} \mathrm{~s}^{-1}$ surface easterlies (in the far field, away from the terrain features; Fig. 1b), one has calm surface winds (Fig. 1c), and the other has $4 \mathrm{~m} \mathrm{~s}^{-1}$ surface westerlies (Fig.1d). All three wind profiles have a 0-6 km shear vector magnitude (a measure of what is sometimes referred to as bulk shear) of $31.8 \mathrm{~m} \mathrm{~s}^{-1}$ and storm-relative helicity (SRH) of $172 \mathrm{~m}^{2} \mathrm{~s}^{-2}$. The problem we are studying is not Galileaninvariant, which adds extra dimensions to the parameter space (this is generally the case when one wishes to include the effects of the lower boundary, e.g., when surface fluxes and/or sloping terrain are included). What will be regarded as the upslope and downslope sides of the terrain will depend on the location of the hodograph trace relative to the origin of the hodograph [i.e., for the hodograph that has surface westerlies (easterlies), the downslope side of a hill is the east (west) side, and the upslope side is the west (east) side].

The environments are horizontally homogeneous at the start of the simulations. Over roughly the first hour of the simulations, the interaction between the base state wind field and terrain results in standing gravity waves and horizontal heterogeneity in the wind and thermodynamic fields (to be discussed further in Section 3). It is this terrain-induced heterogeneity that has a leading-order effect on the convective storms that subsequently pass over the terrain features.

Storms are initiated with an ellipsoidal warm bubble having a maximum potential temperature perturbation of $2 \mathrm{~K}$. The bubble has horizontal and vertical radii of $10 \mathrm{~km}$ and $1.5 \mathrm{~km}$, respectively, over which the perturbation decreases to zero. The bubble is centered $1.5 \mathrm{~km}$ above the ground and 65$125 \mathrm{~km}$ upstream (west) of the terrain features so that the storms would pass over them at approximately $t=2 \mathrm{~h}$ in the simulations presented herein. We performed numerous additional simulations in which the timing of the terrain-crossing was varied from $t=1 \mathrm{~h}$ to $t=3 \mathrm{~h}$; the results reported herein are only those that are robust and do not depend on the exact timing of the passage of the storms over the terrain features. Control simulations also were conducted with flat terrain (described in the next section), as were simulations with varying terrain but without the introduction of a warm bubble (described in Sections 4 and 5). The latter simulations were important for revealing the approximately steady-state wave motions induced by the airflow over the terrain.

\section{Control simulations}

It is necessary to describe the behavior of the simulated supercells occurring over flat terrain before proceeding with the results of the simulations in which terrain variations were 
(a)
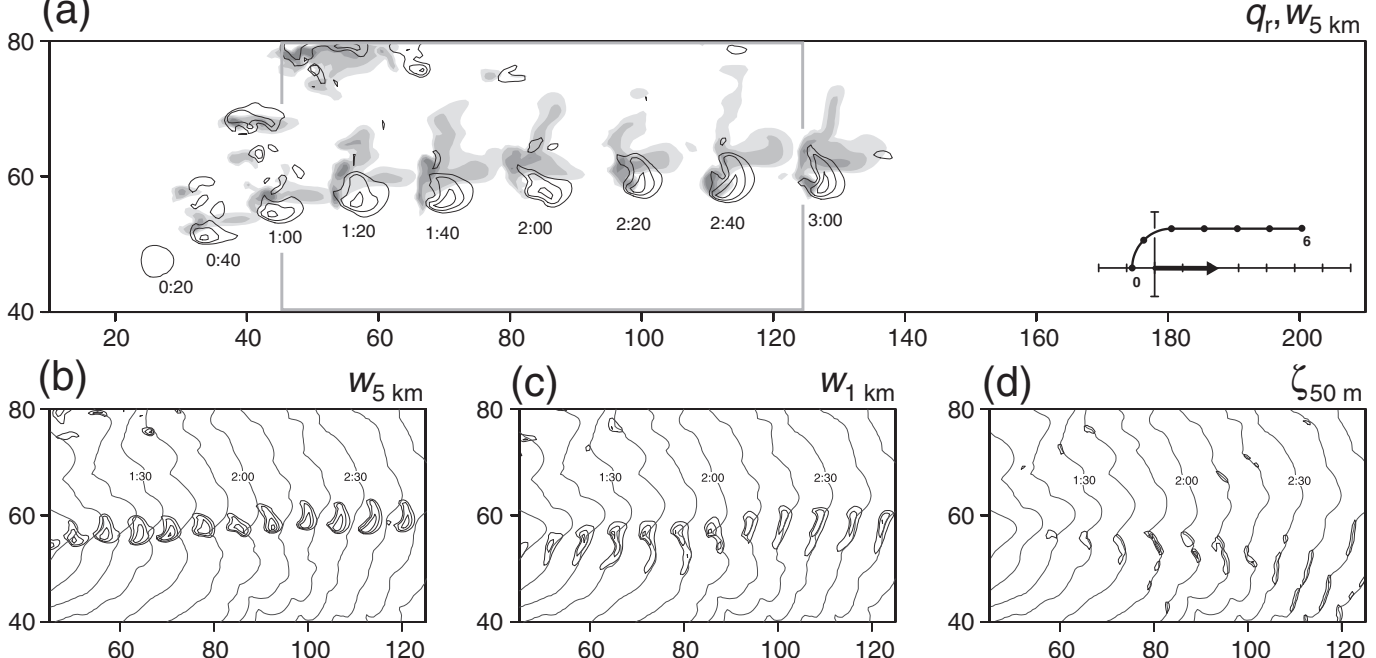

(e)
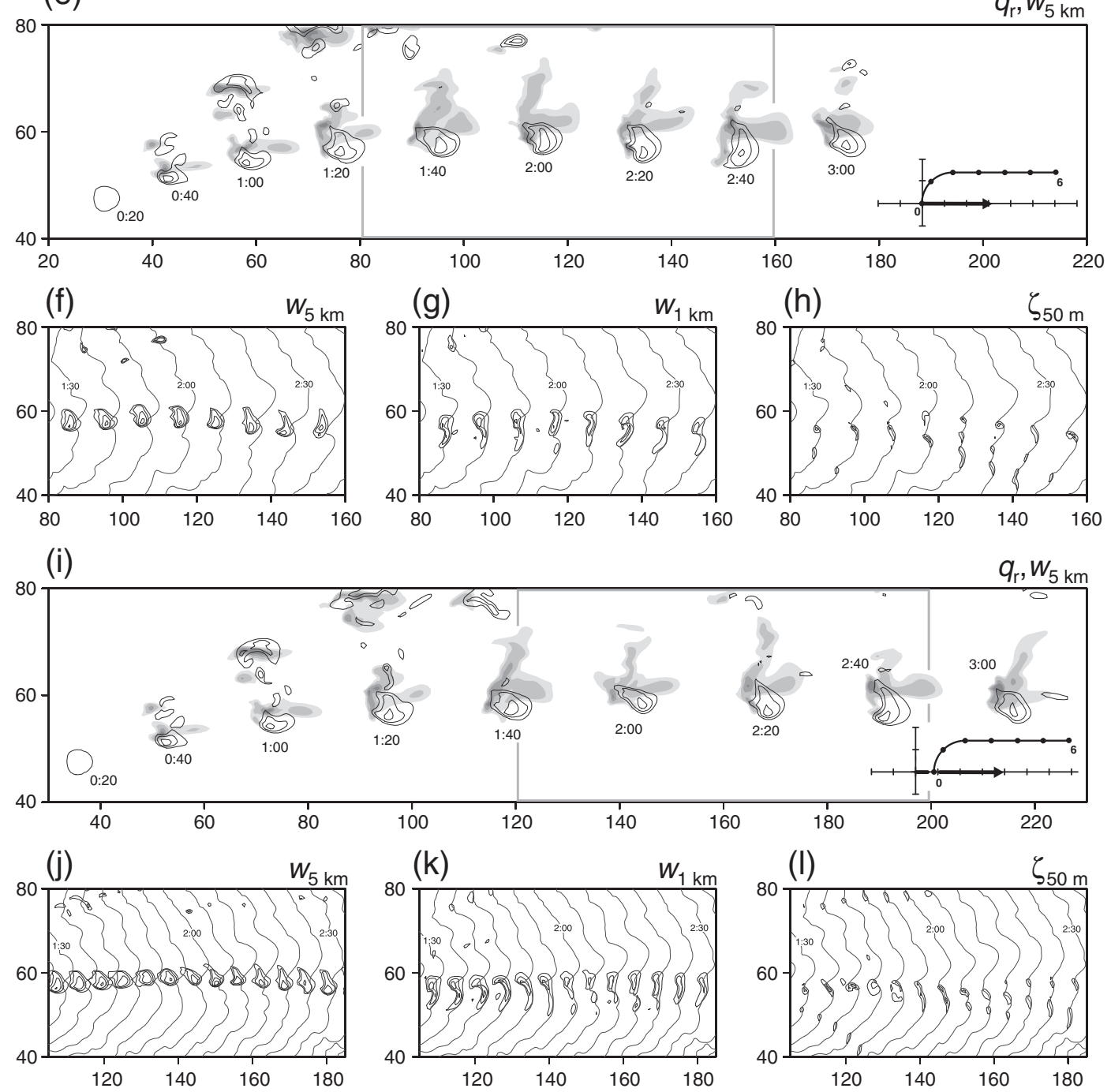
imposed, so that meaningful comparisons between the simulations with and without terrain variations can be made later on. Fig. 2 displays the rainwater (at $z=1 \mathrm{~km}$ ), vertical velocity (at $z=1 \mathrm{~km}$ and $z=5 \mathrm{~km}$ ), and vertical vorticity (at the lowest grid level, i.e., $z=50 \mathrm{~m}$ ) fields of the supercells triggered within the environments characterized by the sounding and hodographs shown in Fig. 1. In the first hour of the control simulations (and also in the simulations with terrain variations), storm-splitting is observed, as also was the case in the Rotunno and Klemp (1982) simulations, which used the same hodograph. The attributes of the hodograph (i.e., the magnitude of the vertical wind shear and the curvature of the hodograph) give rise to a dominant, right-moving (with respect to the mean wind) supercell with cyclonic updraft rotation in every simulation (again, in both flat-terrain and variable-terrain simulations). An approximately steady state is achieved by the right-moving supercell by $t=70 \mathrm{~min}$; the maximum vertical velocity and vertical vorticity are $60-70 \mathrm{~m} \mathrm{~s}^{-1}$ and $0.03-0.05 \mathrm{~s}^{-1}$, respectively, from $t=70 \mathrm{~min}$ through $t=3 \mathrm{~h}$ (not shown). Each simulation also has a weaker, left-moving storm with anticyclonic updraft rotation. The right-moving supercell moves approximately due east in each simulation (its forward speed depends on the position of the hodograph trace relative to the origin of the hodograph, i.e., the groundrelative winds). In the simulations with two-dimensional terrain, the supercell crosses terrain-height contours at very nearly a right angle (again, all of the two-dimensional terrain features in this study are oriented in the north-south direction, such that there are no meridional variations in terrain height). The weaker left-moving storm moves out of the domain to the north.

We also conducted flat-terrain, control simulations with the surface at an elevation $500 \mathrm{~m}$ higher than in the aforementioned control simulations (Fig.3). These simulations will be compared to simulations in which a 500-m escarpment and 500-m-deep valley are introduced (Sections 4.2 and 4.3, respectively). In the escarpment and valley simulations, the flat terrain over which the storm matures before encountering the escarpment and valley (to be discussed further in Section 4), respectively, is at an elevation of $500 \mathrm{~m}$. The surface pressure in these high-altitude control simulations is $946 \mathrm{hPa}$ (versus $1000 \mathrm{hPa}$ in the lower-altitude control simulations). The sounding and wind profiles are the same as those shown in Fig.1, except that the lowest $500 \mathrm{~m}$ of the temperature, moisture, and wind profiles is omitted. Thus, the moist layer is a bit shallower in these simulations than in the ones initialized with the sounding in Fig. 1a, and the wind profiles are characterized by slightly less bulk wind shear and SRH. Moreover, CAPE (CIN) is slightly larger (smaller) in the highaltitude control simulation environment, because the Weisman and Klemp (1982) analytic sounding prescribes environmental potential temperatures that increase monotonically with height (albeit slowly with height at low levels), whereas the water vapor concentration is constant over roughly the lowest $1.5 \mathrm{~km}$ (Fig. 1). The evolution of the supercells in the highaltitude control simulations is qualitatively similar to the evolution of the low-altitude control simulations (cf.Figs. 2 and 3). The supercells in the high-altitude control simulations move slightly $\left(\sim 1 \mathrm{~m} \mathrm{~s}^{-1}\right)$ slower than those in the low-altitude control simulations.

Theoretically, in the case of no surface drag, supercell dynamics are independent of the mean wind velocity and only depend on the shape and length of the hodograph, which define the storm-relative wind profile. However, it is apparent from Figs. 2 and 3 that the supercell simulations using different ground-relative (and therefore grid-relative) wind profiles diverge over time (the warm bubbles that triggered the storms were released from identical positions relative to the scalar gridpoints). The differences among the simulations are purely numerical. Not only does the Courant number vary from simulation to simulation, but storms moving at different grid-relative speeds are sampled differently by the model grid at each time step. Further discussion of this issue is beyond the scope of this paper, as we will be mainly interested in comparing the control simulation for a given hodograph to simulations in which terrain variations are imposed.

\section{Simulations with two-dimensional terrain}

\subsection{0-m tall, 20-km wide hill}

A meridionally oriented hill is placed in the domain in the first suite of simulations with terrain variations (Figs.4-6). The hill is centered at $x=x_{0}$, where $x_{0}=85,120 \mathrm{~km}$, and $145 \mathrm{~km}$ in the simulations in which the surface wind on the hodograph is shifted to the left of the origin (Fig. 1b), is located on the origin (Fig. 1c), and is shifted to the right of the origin (Fig.1d), respectively. The placement of the hill is such that the right-moving (i.e., eastward-moving) supercell crosses the crest at approximately $t=2 \mathrm{~h}$.

The terrain height, $h(x)$, is given by the following "Witch of Agnesi" profile:

$h(x)=\frac{h_{0}}{1+\left(\frac{x-x_{0}}{a}\right)^{2}}$.

The height of the hill, $h_{0}$, is $500 \mathrm{~m}$, and the half-width, $a$, is $10 \mathrm{~km}$, which is the distance from the crest to where the terrain height is half the height of the crest.

Fig. 2. Model output for the flat-terrain, low-altitude control simulations initialized with the wind profiles shown in (a)-(d) Fig.1b, (e)-(h) Fig.1c, and (i)-(l) Fig. 1d. (a),(e), and (i) Rainwater ( $q_{r}$; shaded) and vertical velocity ( $w$; black contours) fields at $z=1 \mathrm{~km}$ and $z=5 \mathrm{~km}$, respectively, at 20-min intervals (times are indicated in $\mathrm{h}$ :mm format). The shading levels for the rainwater fields are $1 \mathrm{~g} \mathrm{~kg}^{-1}, 2 \mathrm{~g} \mathrm{~kg}^{-1}, 4 \mathrm{~g} \mathrm{~kg}^{-1}, 6 \mathrm{~g} \mathrm{~kg}^{-1}$, and $10 \mathrm{~g} \mathrm{~kg}^{-1}$. The contour levels for the vertical velocity fields are $10,20,30$, and $40 \mathrm{~m} \mathrm{~s}^{-1}$. The units on the axes are in $\mathrm{km}$. The hodographs also are inset; storm motions are indicated by the bold vectors, as are the winds at $z=0 \mathrm{~km}$ (" 0 ") and $z=6 \mathrm{~km}$ ("6"), and tick marks along the axes of the hodograph are every $5 \mathrm{~m} \mathrm{~s}^{-1}$. The gray rectangles in (a), (e), and (i) enclose the regions that are displayed in (b)-(d), (f)-(h), and (j)-(l), respectively. (b), (f), and (j) Vertical velocity at $z=5 \mathrm{~km}$ at 10 -min intervals [5-min intervals in (j)] within the regions enclosed by the gray rectangles in (a), (e), and (i), respectively. Only the 20,25, 30, 35, and $40 \mathrm{~m} \mathrm{~s}^{-1}$ contours are shown. The $-1 \mathrm{~K}$ potential temperature perturbation contour at the lowest grid level $(z=50 \mathrm{~m})$ also is plotted at each time interval; it marks the approximate location of the gust front. (c), (g), and (k) As in (b), (f), and (j), respectively, but for vertical velocity at $z=1 \mathrm{~km}$. Only the 5, 7.5, 10, and $12.5 \mathrm{~m} \mathrm{~s}^{-1}$ contours are shown. (d), (h), (l) As in (b), (f), and (j), respectively, but for vertical vorticity at the lowest grid level $(z=50 \mathrm{~m})$. Only the $0.01,0.03$, and $0.05 \mathrm{~s}^{-1}$ contours are shown. 
(a)

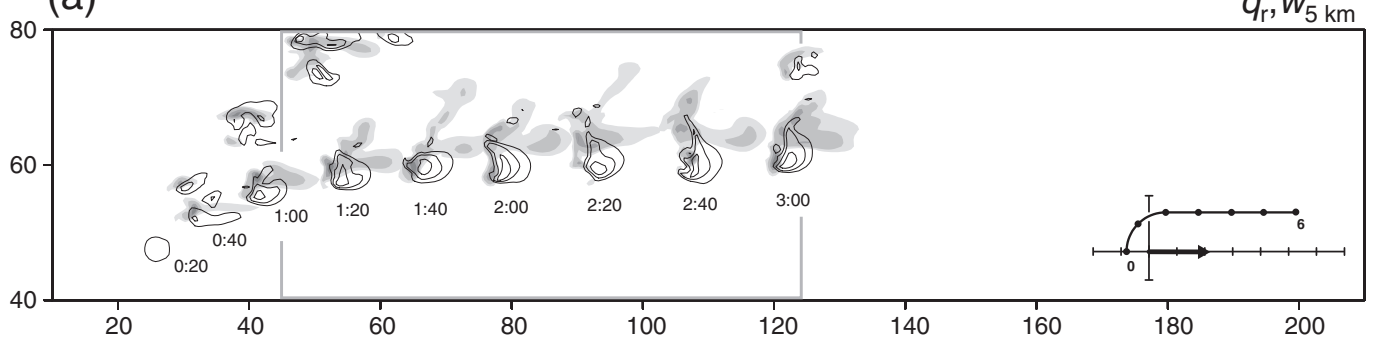

(b)

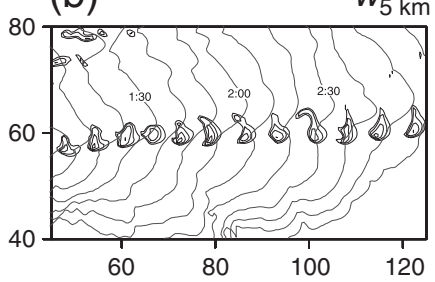

(c)

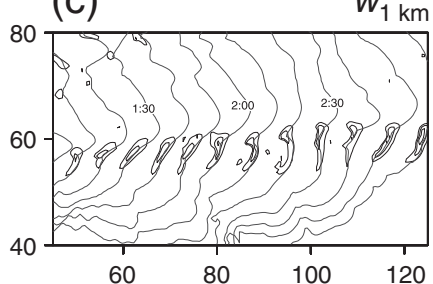

(d)

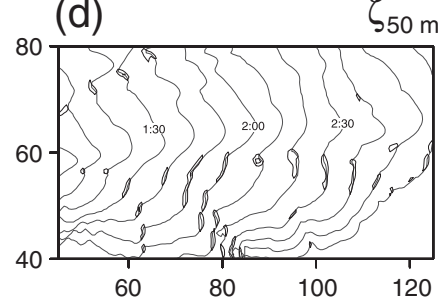

(e)

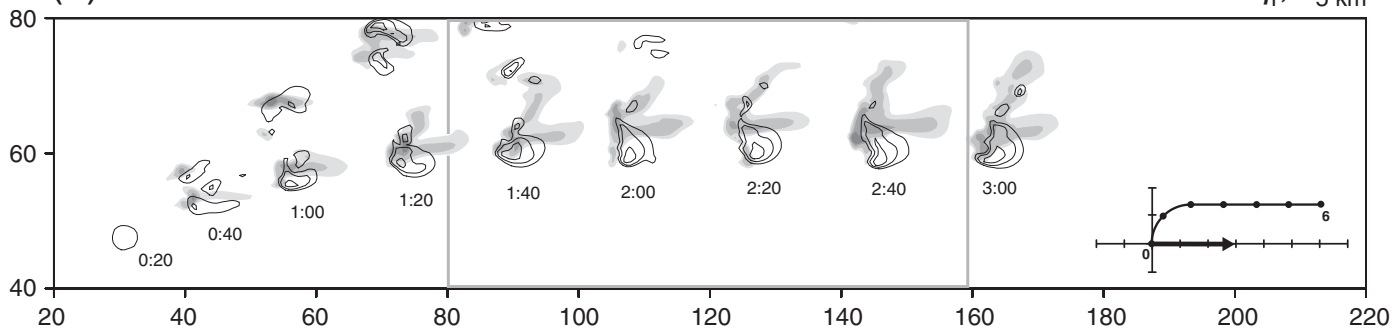

(f)

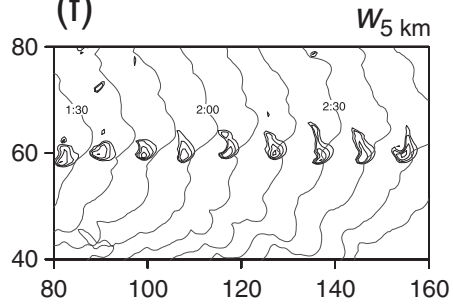

(g)

(h)
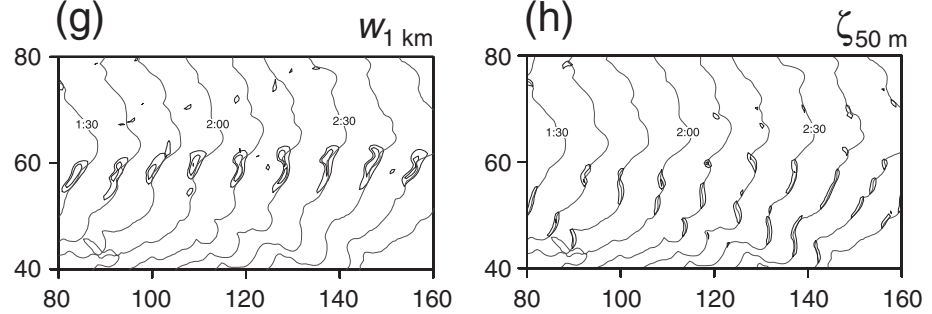

(i)
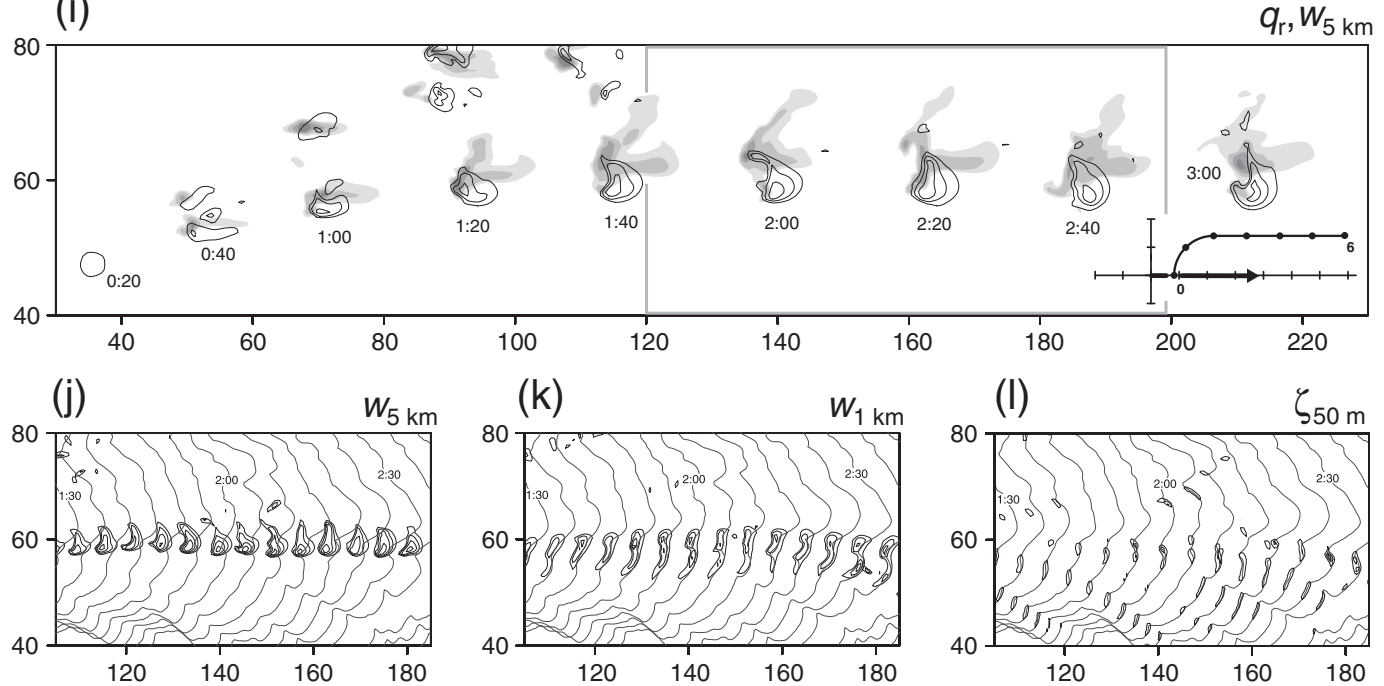

Fig. 3. As in Fig.2, but for the flat-terrain, high-altitude control simulations. The surface is at an elevation of $500 \mathrm{~m}$ (the surface pressure is $946 \mathrm{hPa}$ ). 
(a)
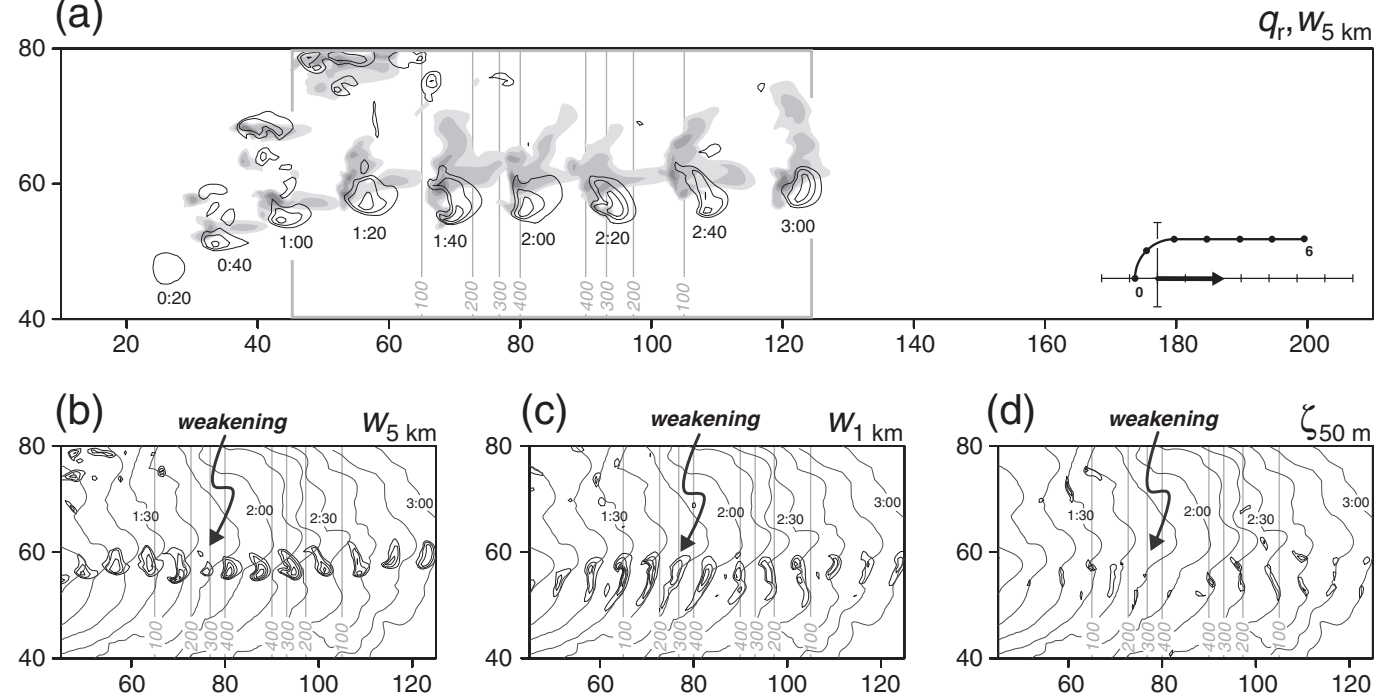

(e)

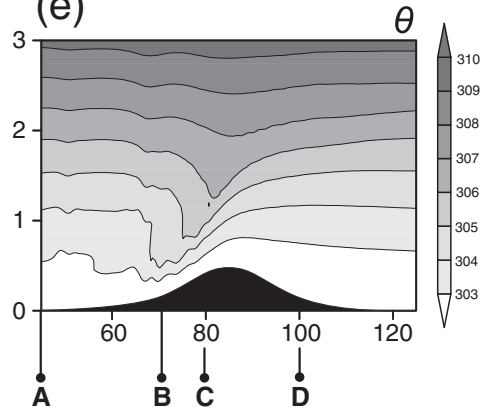

(f)

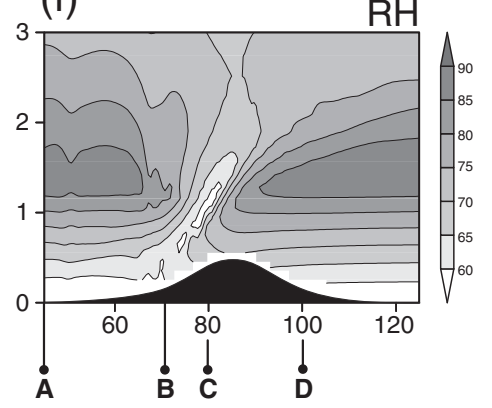

(g)
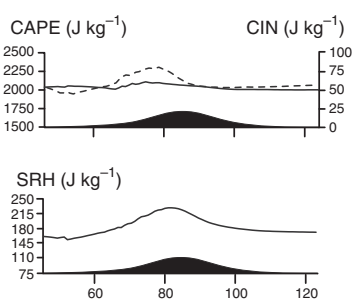

(k)

(j)
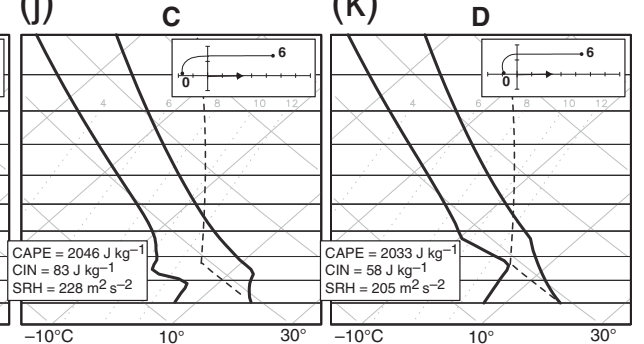

Fig. 4. Model output for simulations for the case of a 500-m tall hill and an environmental wind profile having easterly ground-relative winds at the surface far from the hill (Fig. 1b). (a) Model rainwater ( $q_{r}$; shaded) and vertical velocity ( $w$; black contours) fields at $z=1 \mathrm{~km}$ and $z=5 \mathrm{~km}$ (all heights are above ground level), respectively, at 20-min intervals (times are indicated in h:mm format). The shading levels for the rainwater fields are $1 \mathrm{~g} \mathrm{~kg}^{-1}, 2 \mathrm{~g} \mathrm{~kg}{ }^{-1}, 4 \mathrm{~g} \mathrm{~kg}^{-1}, 6 \mathrm{~g} \mathrm{~kg}^{-1}$, and $10 \mathrm{~g} \mathrm{~kg}^{-1}$. The contour levels for the vertical velocity fields are $10,20,30$, and $40 \mathrm{~m} \mathrm{~s}^{-1}$. The units on the axes are in km. Surface elevations of $100 \mathrm{~m}, 200 \mathrm{~m}$, $300 \mathrm{~m}$, and $400 \mathrm{~m}$ are indicated with gray lines. The hodograph also is inset; the storm motion is indicated by the bold vector, as are the winds at $z=0 \mathrm{~km}$ (" 0 ") and $z=6 \mathrm{~km}$ ("6"), and tick marks along the axes of the hodograph are every $5 \mathrm{~m} \mathrm{~s}^{-1}$. The gray rectangle encloses the region that is displayed in (b), (c), and (d). (b) Vertical velocity at $z=5 \mathrm{~km}\left(w_{5 \mathrm{~km}}\right)$ at 10 -min intervals within the region enclosed by the gray rectangle in (a). Only the 20,25 , 30, 35, and $40 \mathrm{~m} \mathrm{~s}{ }^{-1}$ contours are shown. The $-1 \mathrm{~K}$ potential temperature perturbation contour at the lowest grid level ( $z=50 \mathrm{~m})$ also is plotted at each time interval; it marks the approximate location of the gust front. Surface elevations of $100 \mathrm{~m}, 200 \mathrm{~m}, 300 \mathrm{~m}$, and $400 \mathrm{~m}$ are indicated with gray lines. (c) As in (b), but for vertical velocity at $z=1 \mathrm{~km}$ $\left(w_{1 \mathrm{~km}}\right)$. Only the $5,7.5,10$, and $12.5 \mathrm{~m} \mathrm{~s}^{-1}$ contours are shown. (d) As in (b), but for vertical vorticity at the lowest grid level $\left(z=50 \mathrm{~m} ; \zeta_{50 \mathrm{~m}}\right)$. Only the $0.01,0.03$, and $0.05 \mathrm{~s}^{-1}$ contours are shown. (e) Vertical cross-section of (approximately) steady-state potential temperature ( $\theta$; $\mathrm{K}$ ) in a simulation with the same terrain configuration but without a storm (the units on the vertical axis are in $\mathrm{km}$ ). The region shown is the same region shown in (b)-(d). The letters A, B, C, and D indicate the longitudes of the soundings and hodographs shown in (h)-(k). (f) As in (e), but relative humidity (RH; \%) is displayed. (g) Horizontal profiles of CAPE (solid line), CIN (dashed line), and SRH (solid line) characterizing the (approximately) steady-state environment over the domain shown in (b)-(f). (h)-(k) Select skew $T$-log $p$ diagrams and hodographs depicting the (approximately) steady-state environments at locations A-D [refer to (e) and (f)] in a simulation with the same terrain configuration but without a storm. The CAPE and CIN calculations include the effects of moisture on buoyancy and are based on the pseudoadiabatic ascent of a parcel lifted from the surface (the parcel process curves are indicated with black dashed lines).

The evolution of the convection in the first hour, far west of the hill, closely mirrors the evolution in the flat-terrain simulations described in Section 3. As the right-moving supercells approach the hill, however, small differences in the supercells relative to the control simulations (for all three wind profiles shown in Fig. 1) appear even as early an hour 

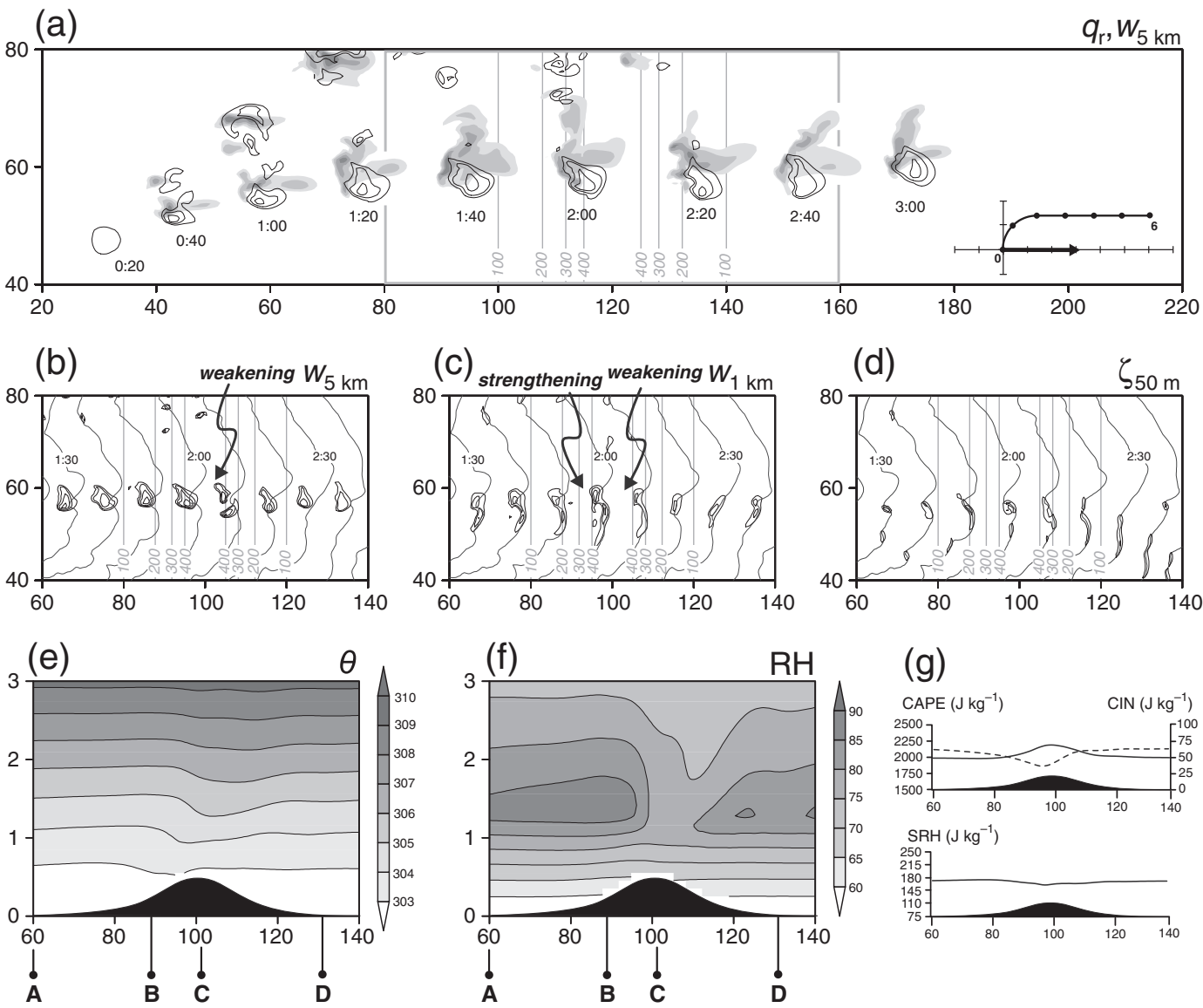

(g)
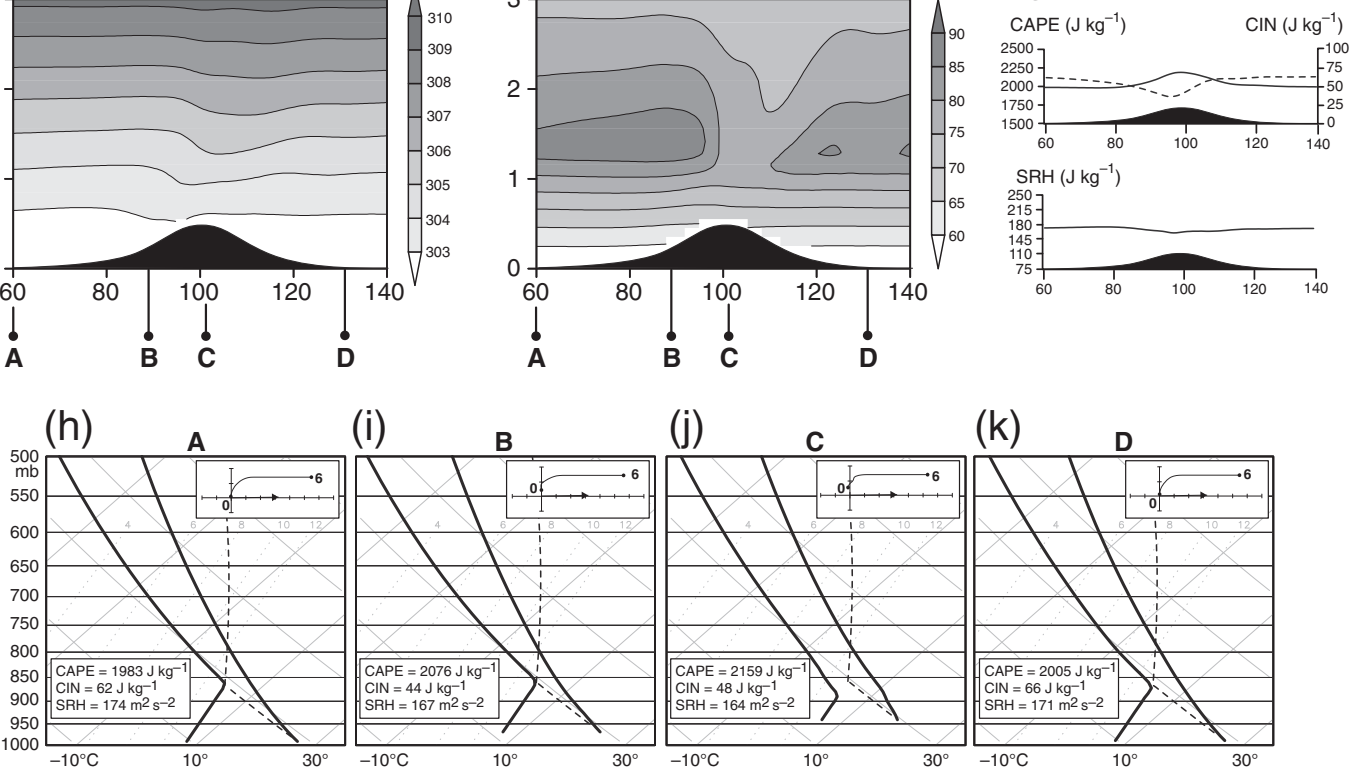

(k)

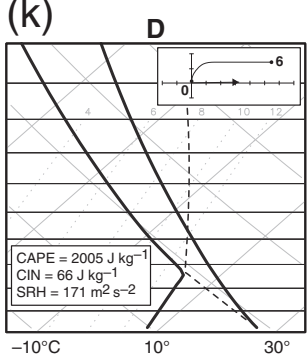

Fig. 5. As in Fig. 4, but for an environmental wind profile having calm ground-relative winds at the surface far from the hill (Fig. 1c).

before (up to $50 \mathrm{~km}$ before) the hill crest is reached, which is well before the terrain slope becomes significant (Figs. 4a, 5a, and 6a; the fields also should be compared to those of the control simulations in Fig. 2a, e, and i). It is impractical to account for every detail in these simulations that differs from the control simulations, especially in the time series of maximum vertical velocity and vertical vorticity (not shown), and these small differences are not robust in the sense that they depend on the exact timing of terrain encounters.

In general, the simulated supercells weaken (in terms of both low-level and midlevel updraft strength and vertical vorticity) on the lee slopes of the hills (Figs.4b-d and 6b-d; cf. Fig. 2b-d and j-1, respectively). [Again, the lee and windward slopes are defined relative to the direction of the surface wind, not the storm motion, for example, in the case of the hodograph trace that is shifted to the left of the origin, there is easterly low-level flow; thus, the eastern (western) slope of the hill is the windward (lee) slope.] The results turn out to be fairly intuitive in that the supercells simply appear to be responding to changes in environmental convective inhibition (CIN) and relative humidity that are induced by the airflow over the terrain. In the lee of the hill, isentropic surfaces ${ }^{1}$ are depressed (Figs.4e and 6e; a hydraulic jump also is present) and relative humidity is anomalously low (Figs.4f and 6f). Both effects contribute to anomalously large CIN on the lee slope (Figs. 4g, i, j and 6g, j). (Figs. 4e-k, 5e-k,

\footnotetext{
${ }^{1}$ The isentropes are streamlines in the case of steady, inviscid, and
} adiabatic flow. 
(a)

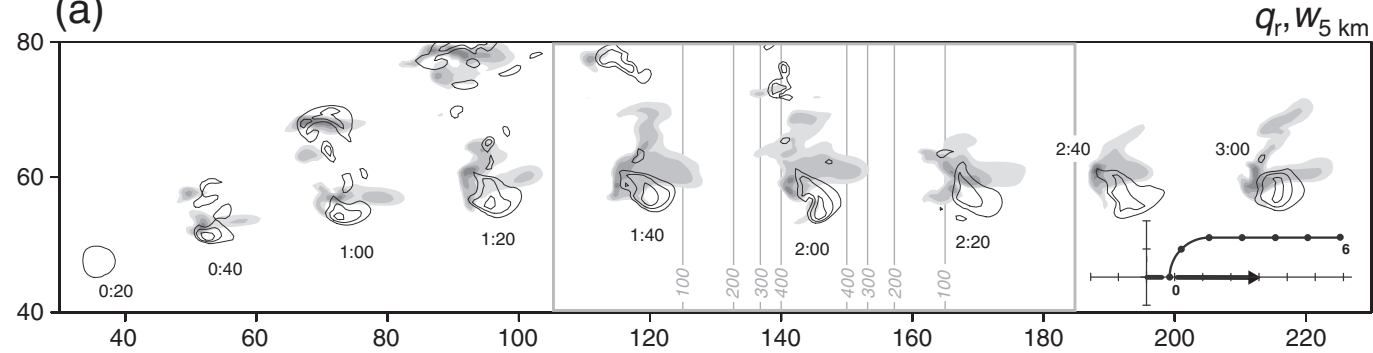

(b) strengthening $\quad W_{5 \mathrm{~km}}$

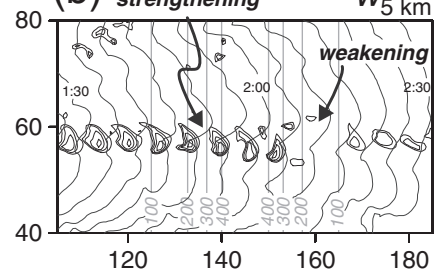

(c)
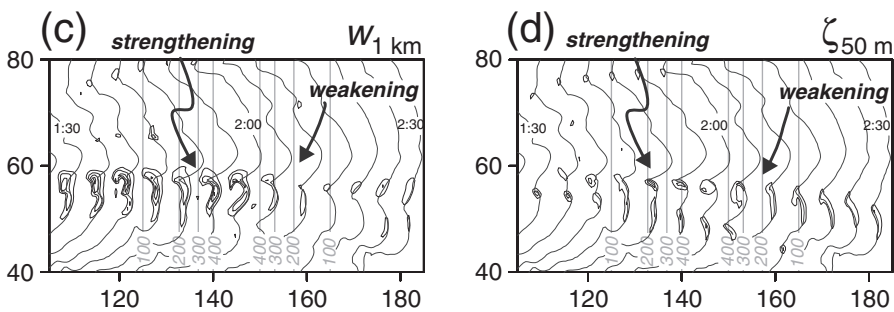

(e)

$\theta$

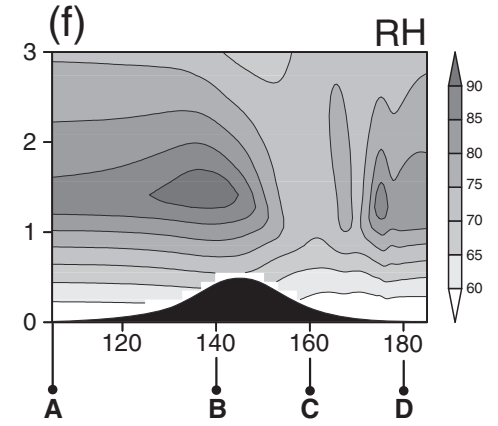

(g)
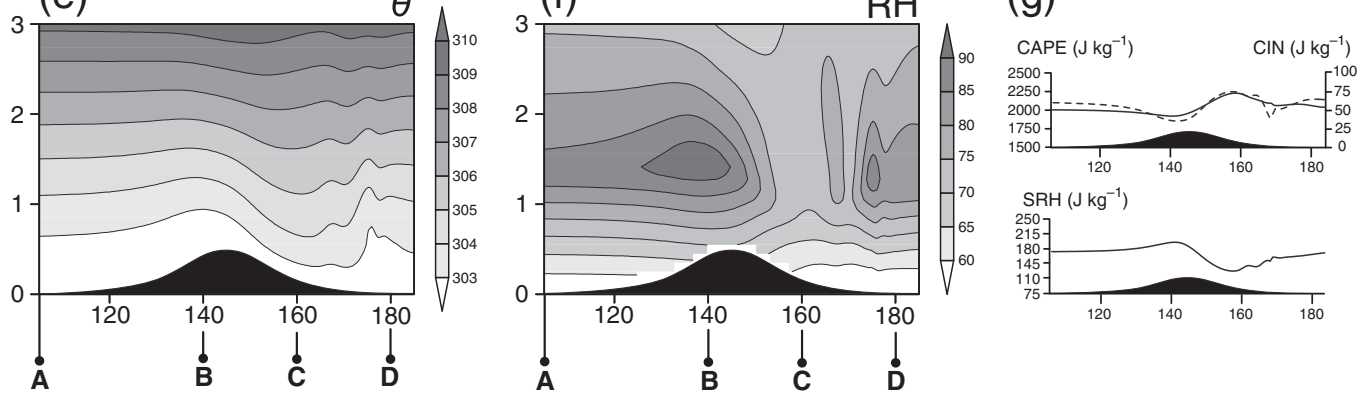

(h)

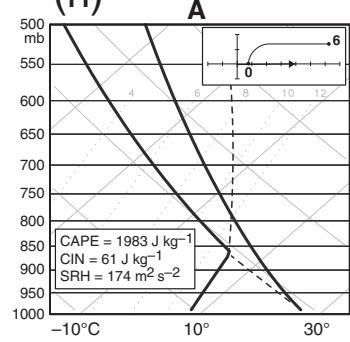

(i)

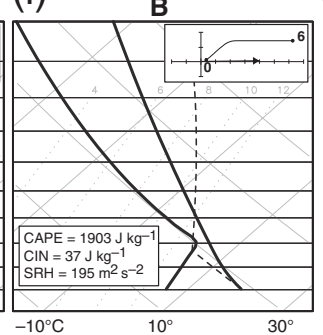

(j)

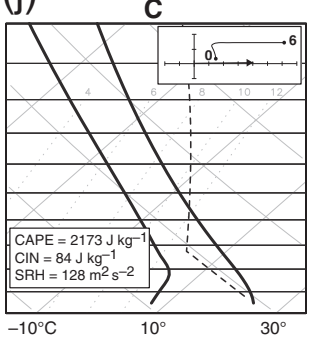

$(\mathrm{k})$

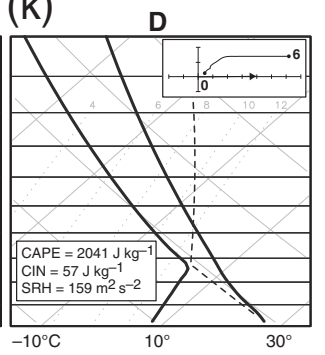

Fig. 6. As in Fig. 4, but for an environmental wind profile having westerly ground-relative winds at the surface far from the hill (Fig. 1d). In (b)-(d), fields are shown every $5 \mathrm{~min}$.

and $6 \mathrm{e}-\mathrm{k}$ are derived from simulations without a storm, that is, simulations in which no warm bubble is introduced at $t=$ 0 , so that the effects of the terrain on the storm environment could be isolated.) Comparison of the soundings on the lee slopes (Figs.4i, j and $6 \mathrm{j}$ ) to the initial sounding (Fig.1a), which is similar to the conditions far upstream of the hill, reveals a decidedly less favorable thermodynamic environment for convection in the lee of the hill in terms of CIN and moisture.

Curiously, even in the simulation initialized with the hodograph having calm surface winds (and negligible up- or downslope wind over the depth of the hill; Fig.1c), the supercell weakens on the east slope (Fig.5b-d) (the east slope cannot really be regarded as the lee slope with respect to the low-level winds given that the cross-hill wind component over the depth of the hill is negligible). Despite the weak low-level flow perpendicular to the hill, the hill still excites standing gravity waves (Fig. 5e), and the motions lead to a relative minimum in relative humidity in the $z=1-3 \mathrm{~km}$ layer from near the crest to the bottom of the eastern slope (Fig. 5f).

In contrast to the aforementioned unfavorable thermodynamic perturbations produced by the terrain, at least for the simulations initialized with hodographs having negligible upor downslope wind over the depth of the hill (Fig.1c) and westerly ground-relative winds at all levels (Fig.1d), there is a modest enhancement of some of the thermodynamic attributes of the environment on the west slope of the hill (the windward slope in the environment depicted in Fig. 1d). In the former environment, the CAPE (CIN) increases 
(decreases) in moving along the west slope of the hill up to the hill crest owing to the higher elevation (as mentioned in Section 3, this is a consequence of the fact that potential temperature increases with height on the Weisman \& Klemp sounding; Fig. $5 g-j$ ), although the CIN reduction is more significant in a relative sense than is the CAPE enhancement. [In general, the relative magnitude of the CAPE perturbations $(1-5 \%)$ is much smaller than the relative magnitude of the CIN perturbations (10-30\%) caused by the hill.] In the latter environment, CAPE and CIN both decrease as the hill crest is approached from the west, although the relative decrease in CIN is much more significant than the relative decrease in CAPE (Fig. 6g-i). The upslope ascent of the environmental air results in windward humidification and a reduction in CIN (notice the upward sloping isentropes and moistening in the relative humidity field on the west slope of the hill in Fig. 6e, f). In both simulations, the supercells exhibit modest strengthening as the hill crest is approached (in the case of the environment with weak hill-relative winds, the strengthening is most apparent in the low-level updraft) before weakening occurs on the eastern slope of the hill (Figs. $5 \mathrm{c}$ and $6 b-d)$. The intensification described here is relative to the control supercells (Fig. $2 \mathrm{f}-\mathrm{h}$ and $\mathrm{j}-\mathrm{l}$, respectively); windward intensification also is observed following lee weakening in the case of easterly low-level ground-relative winds (Figs. 1b and $4 \mathrm{~b}-\mathrm{d}$ ), but the intensification is more of a recovery of the storm to its prior state over flat terrain than the storm becoming more intense than it would have in the absence of a hill.

Not only does the environmental flow over the hill introduce thermodynamic heterogeneity into the storm environment, but it also introduces horizontal heterogeneity in the wind shear. SRH is enhanced (reduced) on the lee slope in the case of easterly (westerly) low-level ground-relative winds (Figs. $4 \mathrm{~g}, \mathrm{i}, \mathrm{j}$ and $6 \mathrm{~g}, \mathrm{j}$, respectively). In the case of easterly low-level ground-relative winds, the possible enhancing effect of the increased shear on the lee slope is apparently outweighed by the detrimental effects of the increased CIN and/or decreased relative humidity. In the case of the hodograph with negligible up- or downslope wind over the depth of the hill (Fig.1c), the hill does not introduce significant horizontal heterogeneity in the vertical wind shear fields (Fig. $5 g-k$ ).

\subsection{0-m tall, 10-km wide escarpment}

In the next set of simulations, a meridionally oriented escarpment separates two flat regions that differ in altitude by $500 \mathrm{~m}$, with the higher (lower) terrain being to the west (east). The terrain height is given by

$$
h(x)= \begin{cases}h_{0}, & x \leq x_{0} \\ h_{0}+\left(\frac{h_{1}-h_{0}}{x_{1}-x_{0}}\right)\left(x-x_{0}\right), & x_{0}<x<x_{1} \\ h_{1}, & x \geq x_{1}\end{cases}
$$

where $h_{0}$ and $h_{1}$ are the elevations of the flat terrain west and east of the escarpment, respectively, and the terrain drops from $h_{0}$ to $h_{1}$ over $x_{0}<x<x_{1}$. The variables have the following values: $h_{0}=500 \mathrm{~m}, h_{1}=0 \mathrm{~m}, x_{0}=80,115$, and $140 \mathrm{~km}$ for the hodographs in Fig. $1 \mathrm{~b}-\mathrm{d}$, respectively, and $x_{1}=90,125$, and $150 \mathrm{~km}$ for the same respective hodographs. Thus, the drop-off in elevation from $500 \mathrm{~m}$ to $0 \mathrm{~m}$ occurs over a horizontal distance of $10 \mathrm{~km}$.

As might be expected in light of the results of the simulations containing a hill, the airflow over the escarpment introduces horizontal heterogeneity in the storm environment (Figs.7-9). The horizontal heterogeneity is much greater in the case of westerly low-level, ground-relative winds (Fig.9e-k) than in the case of easterly low-level, ground-relative winds (Fig.7e-k) and negligible cross-escarpment, low-level winds (Fig. 8e-k).

In the case of westerly low-level, ground-relative (downslope) winds, the isentropic surfaces have two major dips in the lee of the escarpment (Fig. 9e), which are also regions of reduced relative humidity (Fig.9f) and enhanced CIN (Fig.9g, i, j). The low-level shear and SRH are also greatly reduced in the lee of the escarpment (Fig.9g, i, j). Thus, the lee of the escarpment, in the case of westerly ground-relative winds, is a decidedly less favorable storm environment than either the far-field environment on the high terrain (Fig.9h) or the far-field environment on the low terrain (Fig.9k). As a result, the supercell's updraft weakens dramatically upon reaching the escarpment (Fig.9b, c; cf. Figs. $2 \mathrm{j}$, k and 3j, k). The low-level vertical vorticity maximum weakens as well, but to a lesser degree (Fig. 9d; cf. Figs. 2l and 31).

In the cases of easterly low-level, ground-relative (upslope) winds (Fig.1b) and negligible cross-escarpment, low-level winds (Fig. 1c), midlevel updraft strength does not change appreciably as the storms cross the escarpment (Figs.7b and 8b). However, the low-level updrafts briefly strengthen immediately west of the escarpment and then weaken as the storms descend the escarpment (Figs.7c and $8 \mathrm{c}$ ). The low-level updrafts regain their pre-escarpment intensity by the time they are $\sim 20 \mathrm{~km}$ east of the escarpment. The evolution of the low-level updraft cannot be attributed simply to changes in the CIN, relative humidity, or shear, as there is nothing obviously favorable (hostile) about the environment, relative to the far field, in the region of lowlevel updraft strengthening (weakening) (cf. Fig.7h-j; cf. Fig. $8 \mathrm{~h}-\mathrm{j}$ ), nor is it known why only the low-level updraft evolves in this manner. A full exploration of this aspect of the simulations is outside of the scope of the present paper. Lastly, the low-level vertical vorticity maximum does not undergo any significant changes in intensity relative to the control simulations upon crossing the escarpment (Figs.7d and 8d; cf. Figs. 2d, h and 3d, h).

\subsection{0-m deep, $15-\mathrm{km}$ wide valley}

In the next set of simulations, we introduce a meridionally oriented valley, $500-\mathrm{m}$ deep and $15-\mathrm{km}$ wide. The terrain height is given by

$$
h(x)= \begin{cases}h_{0}, & x \leq x_{0} \\ h_{0}+\left(\frac{h_{1}-h_{0}}{x_{1}-x_{0}}\right)\left(x-x_{0}\right), & x_{0}<x<x_{1} \\ h_{1}, & x_{1} \geq x \leq x_{2} \\ h_{1}+\left(\frac{h_{2}-h_{1}}{x_{3}-x_{2}}\right)\left(x-x_{2}\right), & x_{2}<x<x_{3} \\ h_{2}, & x \geq x_{3}\end{cases}
$$


(a)

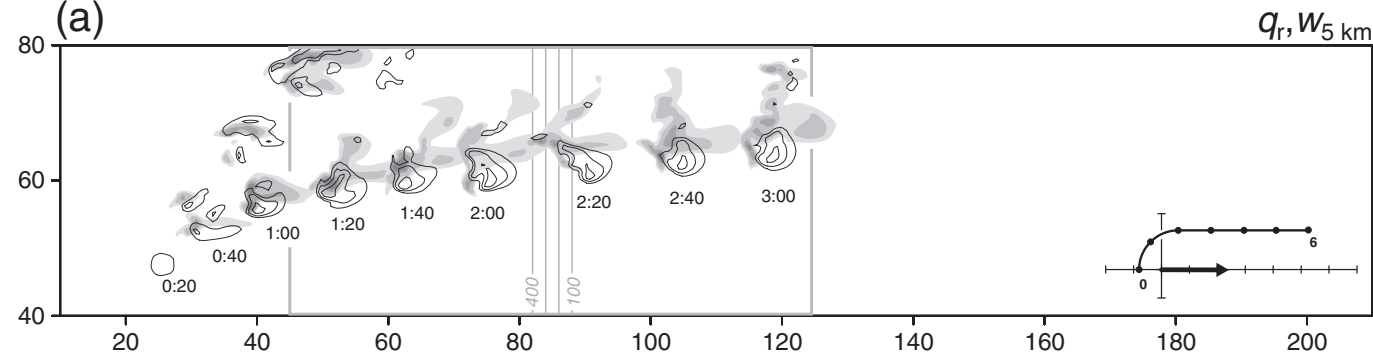

(b)

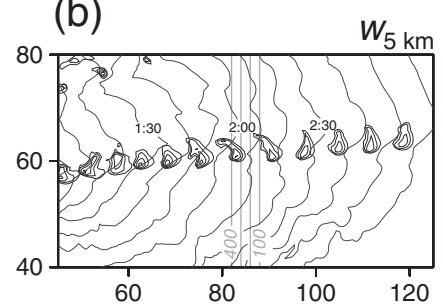

(e)

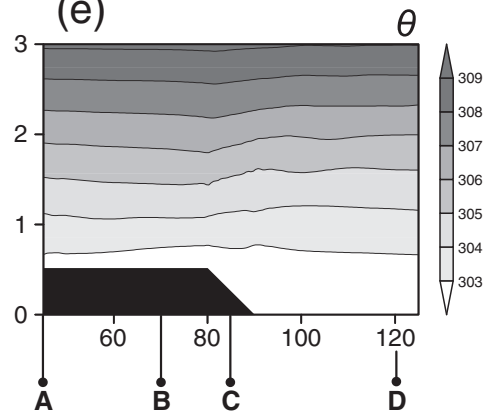

(c)

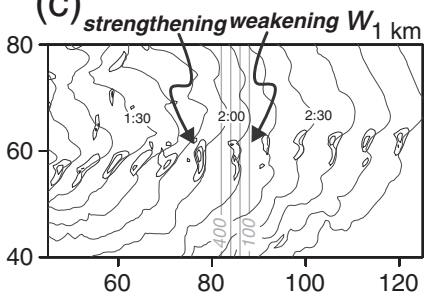

(f)

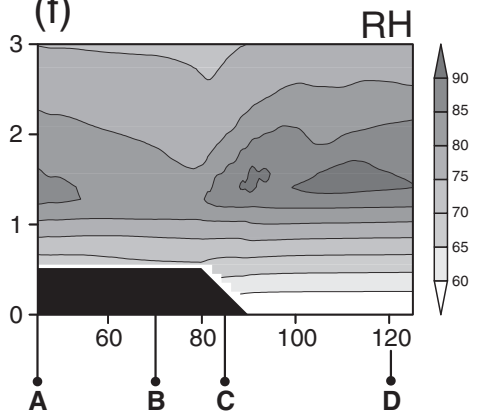

(d)

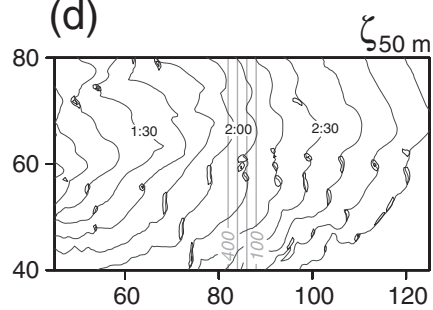

(g)
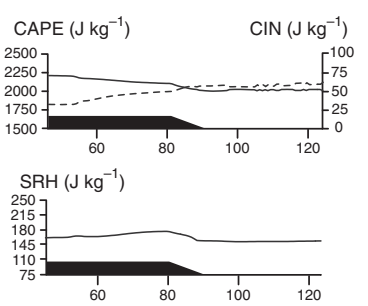

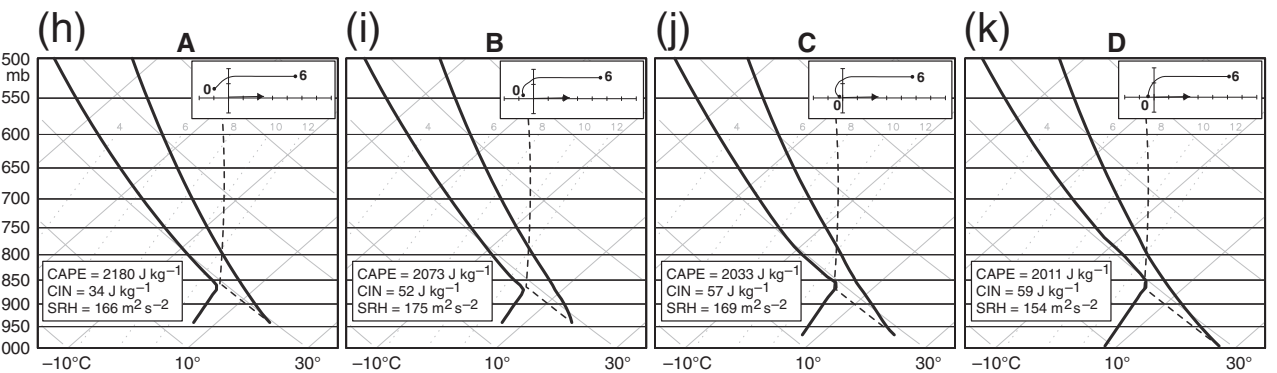

Fig. 7. As in Fig. 4, but for the case of a 500-m tall escarpment.

where $h_{0}$ and $h_{2}$ are the elevations of the flat terrain west and east of the valley, respectively, $h_{1}$ is the elevation of the valley, the terrain drops from $h_{0}$ to $h_{1}$ over $x_{0}<x<x_{1}$, and the terrain rises from $h_{1}$ to $h_{2}$ over $x_{2}<x<x_{3}$. The variables have the following values: $h_{0}=h_{2}=500 \mathrm{~m}, h_{1}=0 \mathrm{~m}, x_{0}=80,115$, and $140 \mathrm{~km}$ for the hodographs in Fig. 1b-d, respectively, and, for the same respective hodographs, $x_{1}=90,125$, and $150 \mathrm{~km}, x_{2}=105,140$, and $165 \mathrm{~km}$, and $x_{3}=115,150$, and $175 \mathrm{~km}$. Thus, the valley is $15 \mathrm{~km}$ wide, the drop-off in elevation from $500 \mathrm{~m}$ to the valley floor $(0 \mathrm{~m})$ occurs over a horizontal distance of $10 \mathrm{~km}$, and the rise in elevation from the valley floor to the high terrain east of the valley also occurs over a horizontal distance of $10 \mathrm{~km}$.

The presence of two locations from which terraingenerated gravity waves can originate, that is, the longitude where the terrain drops into the valley and, farther east, the longitude where the terrain abruptly rises again, greatly complicates things owing to wave interactions. The environmental potential temperature and relative humidity fields in these simulations are the most complex of the two-dimensional terrain simulations we investigated (Figs. 10e, f,11e, f, and 12e, f). Though the details are sensitive to the width of the valley (only the results for a $15-\mathrm{km}$ wide valley are presented herein), in general, the simulated storms, upon crossing into the valley, behave in a manner similar to the behavior observed when storms pass over an escarpment, as described in Section 4.2 (Figs.10b-d,11b-d, and 12b-d). That is, updrafts weaken upon crossing from higher terrain to lower terrain, regardless of the low-level, ground-relative wind velocity. There are some curious differences, however. For 
(a)

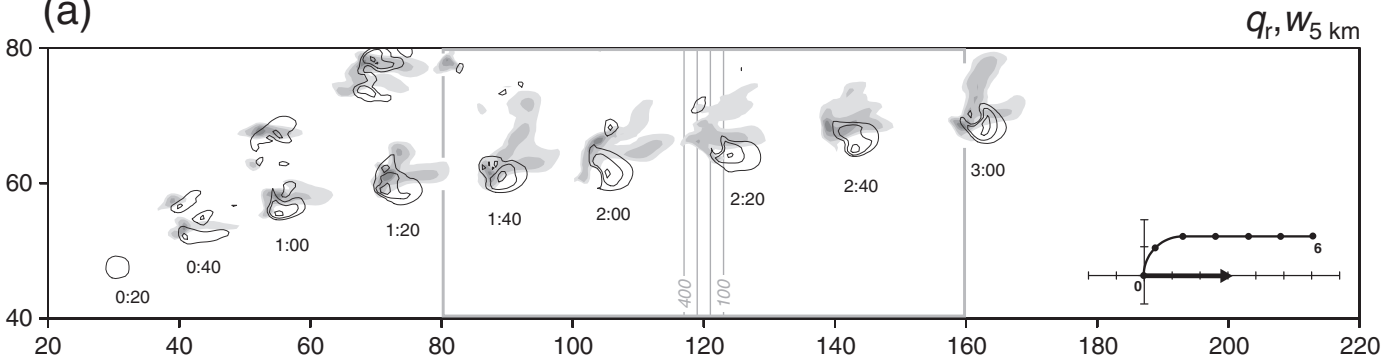

(b)

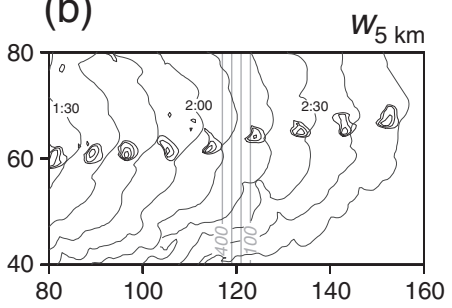

(e)

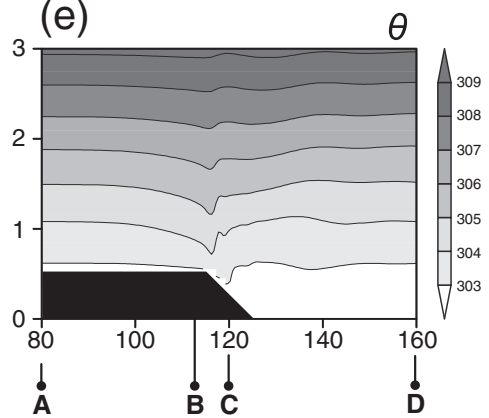

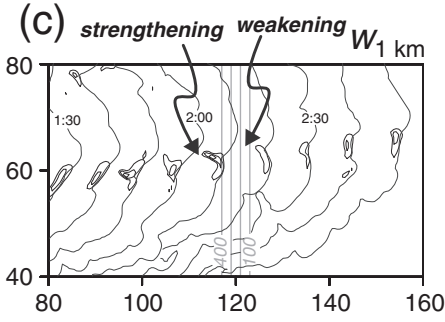

(f)

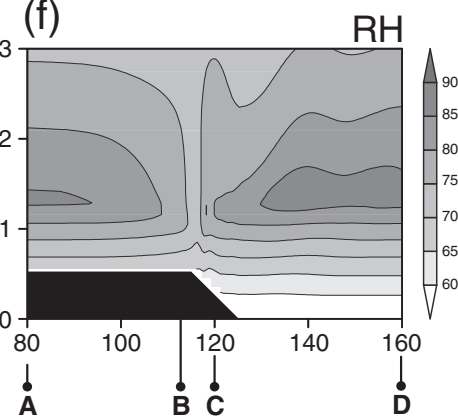

(d)

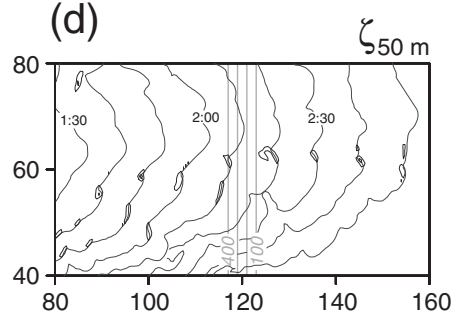

(g)

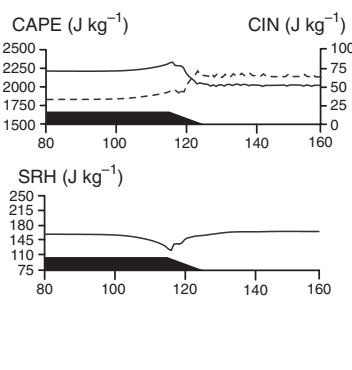

(k)

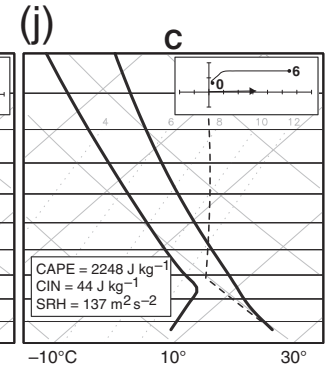

(i)

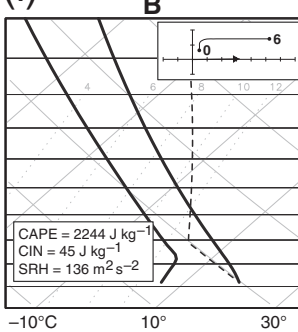

(i)$$
\text { . }
$$

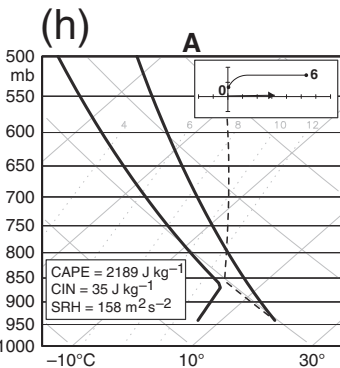

Fig. 8. As in Fig.7, but for an environmental wind profile having calm ground-relative winds at the surface far from the escarpment (Fig. 1c).

example, in the case of easterly low-level ground-relative winds, the low-level updraft weakens much more when the storm enters the valley than when the storm crosses over the escarpment in the simulations of Section 4.2 (cf. Figs.7c and 10c), even though the terrain slope is identical. These differences ultimately must be the result of wave interactions between waves originating on opposite sides of the valley.

The overall tendency is for storms in the valley simulations (for all wind profiles) to have their minimum intensities, as measured by updraft and mesocyclone strengths, over the valley. The storms take a slight left turn during these weak phases, owing to a weakening of the dynamic vertical pressure gradients of the storms, which promote propagation to the right of the mean wind over the depth of the storm. The storms regain their former intensities upon reaching higher ground east of the valley, although in the simulation with easterly low-level ground-relative winds, the storm intensifies as it passes over the upsloping terrain on the east side of the valley, abruptly weakens once it reaches the high terrain, and then ultimately regains its former pre-valley intensity (Fig. 10a-d; this simulation was carried out through $4 \mathrm{~h}$ ). A thorough investigation of this and other oddities observed in the valley simulations, which ultimately are related to complicated wave interactions, will have to await a future study.

\section{Simulations with three-dimensional terrain}

When three-dimensional terrain is introduced, the effect of terrain on the storm environment can be further 
(a)
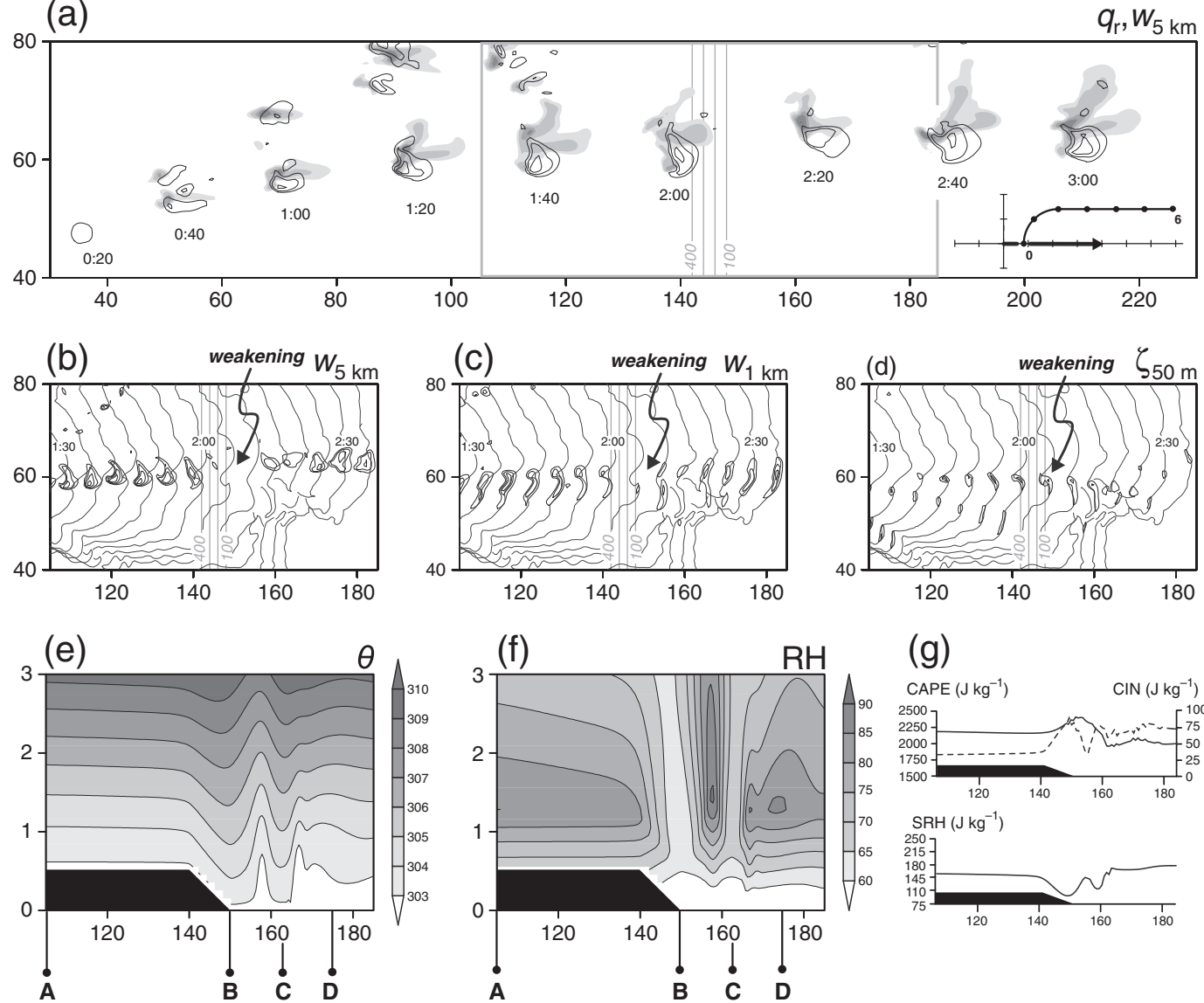

(g)
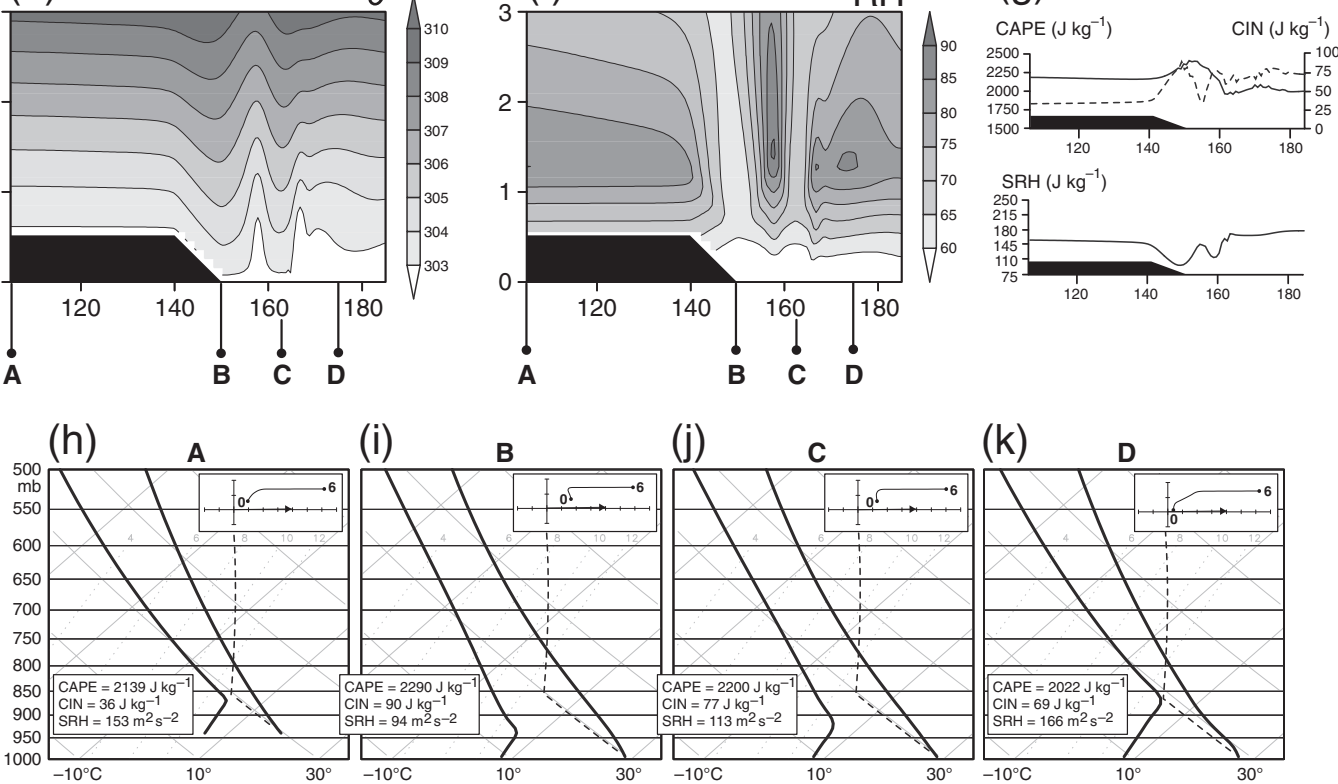

Fig. 9. As in Fig. 7, but for an environmental wind profile having westerly ground-relative winds at the surface far from the escarpment (Fig. 1d). In (b)-(d), fields are shown every $5 \mathrm{~min}$.

complicated by mesoscale vortices that can form in the lee of terrain obstacles. Most mesoscale, terrain-induced, lee vortices are believed to form baroclinically (Smolarkiewicz and Rotunno, 1989; Epifanio and Durran, 2002) rather than as a result of the separation of a viscous boundary layer from an obstacle [wake vortices appear in numerical simulations in which a free-slip lower boundary condition (i.e., no surface drag, purely inviscid) is employed, in fact, the vortices actually weaken as surface friction is increased (Smolarkiewicz and Rotunno, 1989)]. The baroclinic generation of lee vortices requires a stably stratified lower troposphere. More specifically, wake vortices are usually observed in environments characterized by a mountain Froude number (inverse nondimensional mountain height), $\mathrm{Fr}_{\mathrm{m}}=U / N h_{0}$ (where $U$ is the characteristic cross-barrier wind speed and $N$ is the Brunt-Väisälä frequency, and both are evaluated over the depth of the barrier), in the range of 0.1-0.5.

The baroclinic mechanism of vortex generation is briefly explained as follows ${ }^{2}$ : On the upwind side of an obstacle, upsloping winds cause isentropes to bulge upward, resulting in a region of negative buoyancy, horizontal buoyancy gradients, and generation of horizontal vorticity (vortex rings encircle the buoyancy minimum). Horizontal advection of vorticity

\footnotetext{
2 The reader is referred to Smolarkiewicz and Rotunno (1989) and Epifanio and Durran (2002) for a more detailed explanation.
} 
(a)

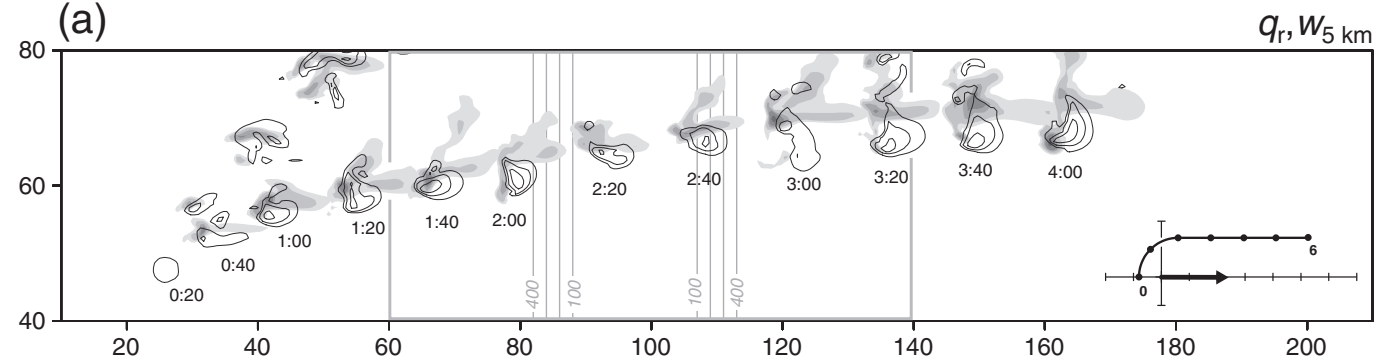

(b)

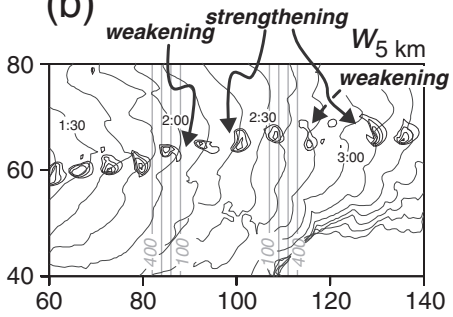

(e)

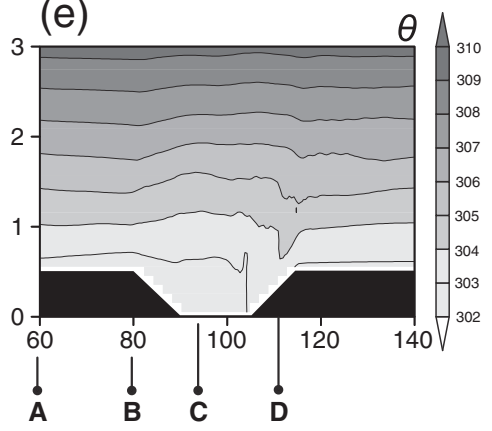

(c)

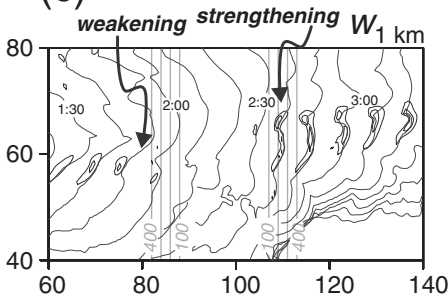

(f)

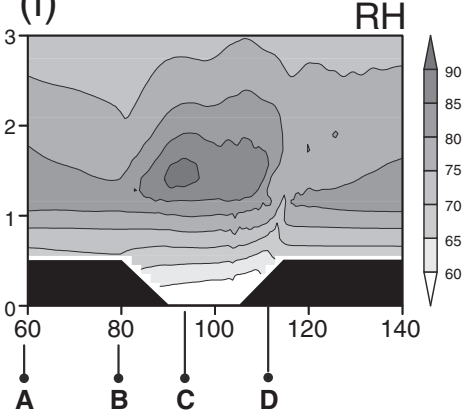

(d)

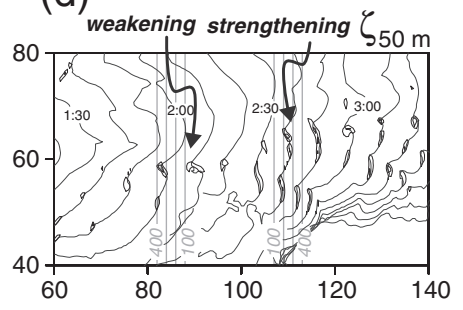

(g)

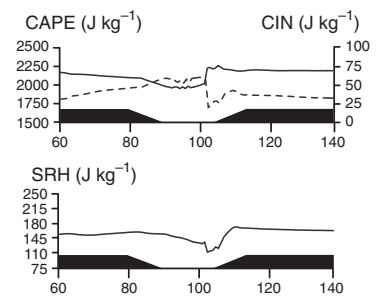

(h)

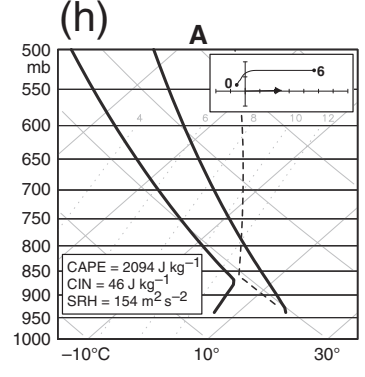

(i)

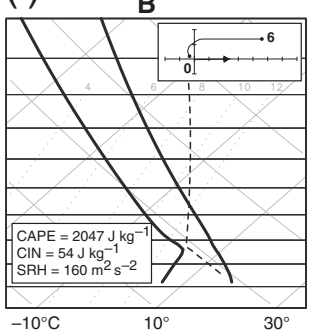

(j)

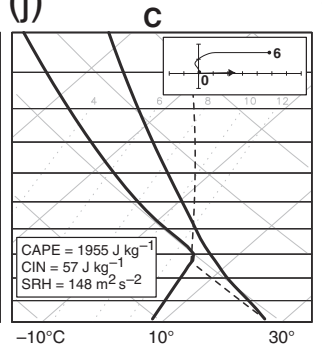

$(\mathrm{k})$

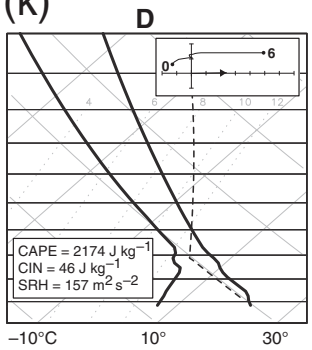

Fig. 10. As in Fig. 4, but for the case of a 500-m deep valley.

displaces the vortex rings downwind from the buoyancy minimum. (In the absence of horizontal advection of vorticity, the vortex rings would be co-located with the buoyancy contours, although this limiting case actually cannot be achieved because the negative buoyancy is a direct result of a horizontal airstream ascending the obstacle in a stably stratified atmosphere.) Furthermore, isentropes bend downward in the wake of the obstacle, leading to a region of positive buoyancy immediately downstream of the obstacle. The horizontal buoyancy gradients associated with the buoyancy maximum generate vortex rings having the opposite sense of rotation as the vortex rings generated upwind as a result of the buoyancy minimum. Like the upwind-generated vortex rings, these vortex rings also are shifted downwind of the warm anomaly owing to horizontal advection. Negative vertical velocities are found downwind of the obstacle, and the interaction of the baroclinically generated vortex rings with the horizontal gradients of vertical velocity on the immediate downstream wide of the obstacle produce vertical vorticity by way of tilting. A pair of counter-rotating vortices straddles the minimum vertical velocity immediately downstream of the obstacle.

In these simulations we only consider the wind profile shown in Fig.1d, that is, the one that possesses the smallest degree of directional wind shear. This is the wind profile that produces the most prominent baroclinically generated vertical vorticity extrema in the lee of the isolated hill and gap. We 
(a)

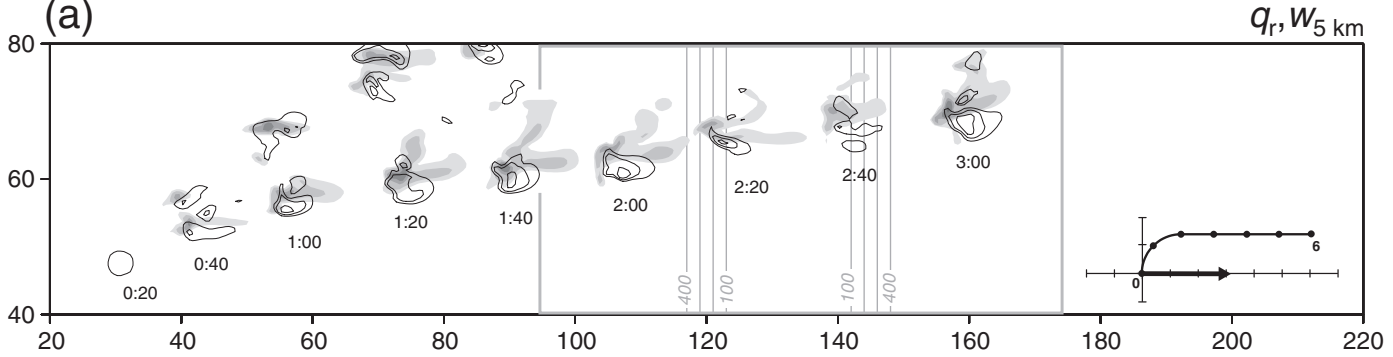

(b)

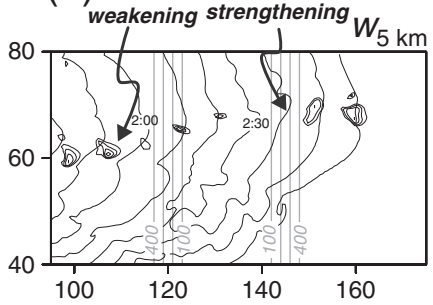

(e)

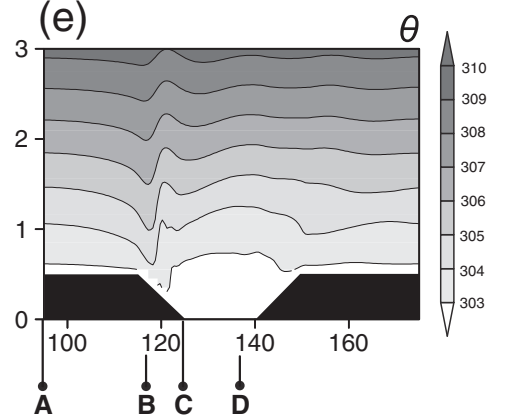

(c)

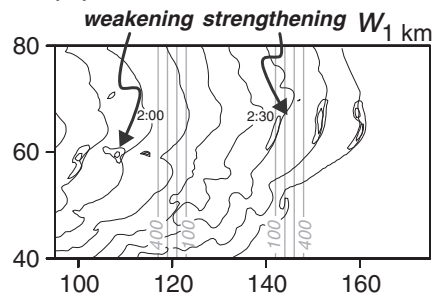

(f)

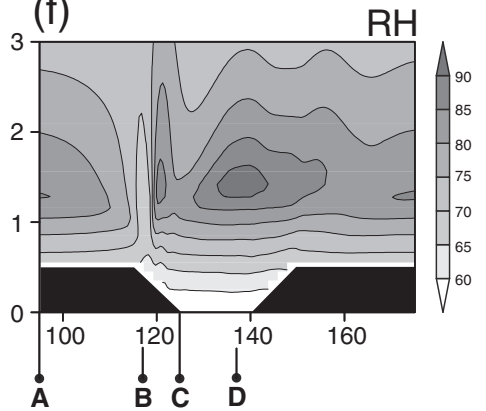

(d)

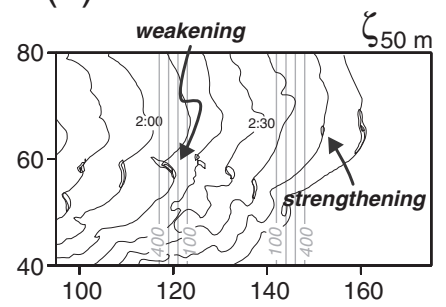

(g)

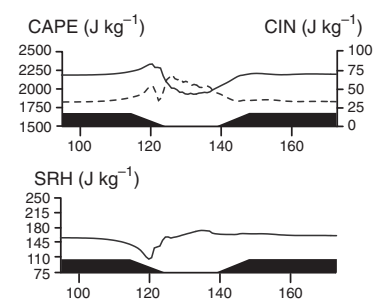

(h)

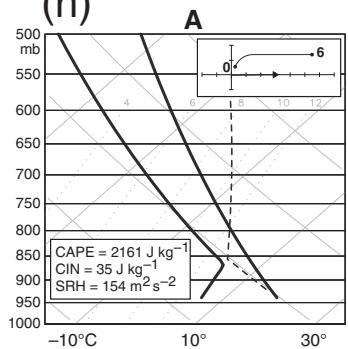

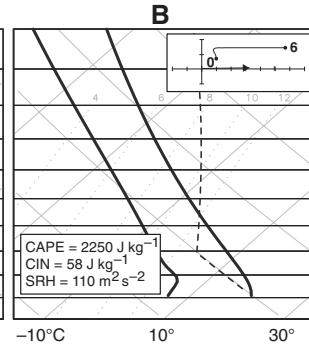
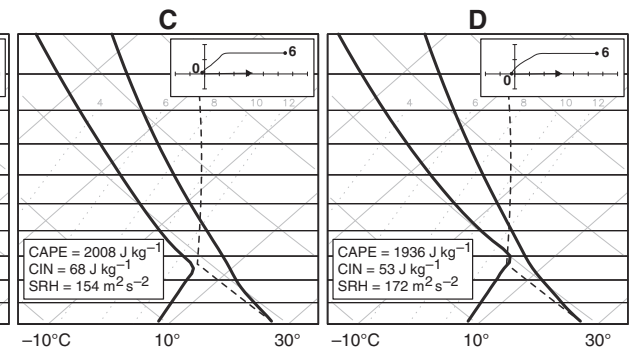

Fig. 11. As in Fig. 10, but for an environmental wind profile having calm ground-relative winds at the surface far from the valley (Fig. 1c).

deliberately identify the terrain-induced vertical vorticity perturbations as extrema rather than vortices because closed streamlines are not observed. The lack of well-defined vortices in our simulations probably is due to the mountain Froude number being larger than optimal in our convective storm environments $\left(U / N h_{0} \approx 1.3\right)$. Nonetheless, the airflow over and around the terrain obstacles still results in the formation of distinct mesoscale vertical vorticity anomalies having a horizontal scale comparable to the horizontal scale of the obstacles. As will be evident below, the upstream wind profile favored cyclonic lee vorticity maxima over anticyclonic vorticity maxima. This ultimately must be a result of the hodograph curvature, which causes the terrain-normal wind component to vary with height, and this variation must have been in a way such that asymmetric lee vorticity anomalies arose, with the cyclonic member dominating (a full examination of the effects of hodograph curvature on the development of lee vortices is beyond the scope of this article). The magnitude of the cyclonic terrain-induced vorticity anomaly is several times the magnitude of the Coriolis parameter in mid latitudes. Moreover, the airflow over the obstacles also introduces horizontal heterogeneity in the CAPE, CIN, and SRH fields as was the case in the simulations with two-dimensional terrain. 


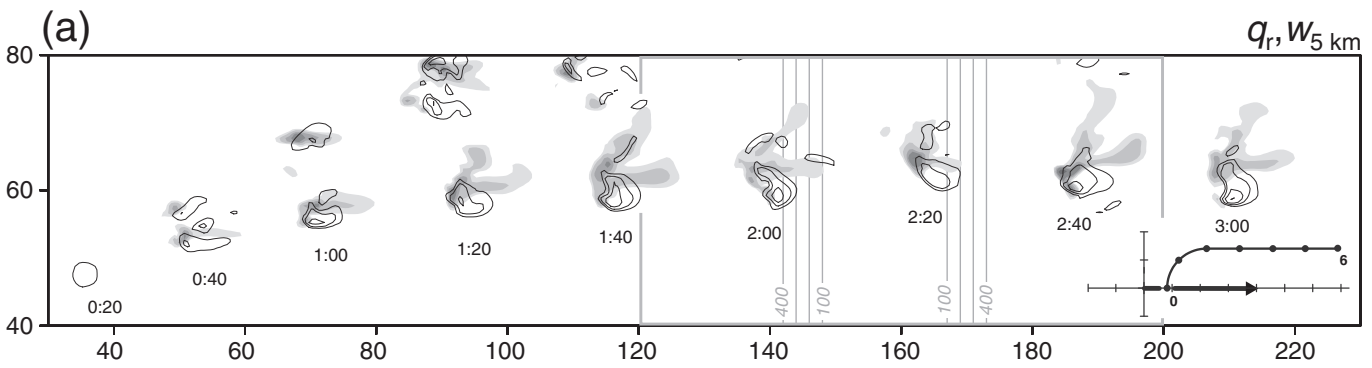

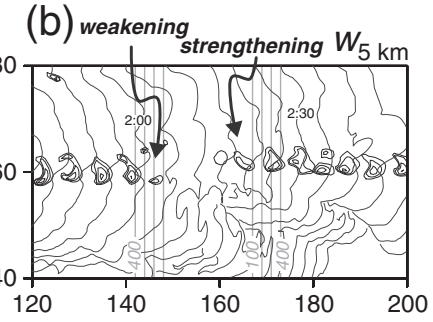

(e)

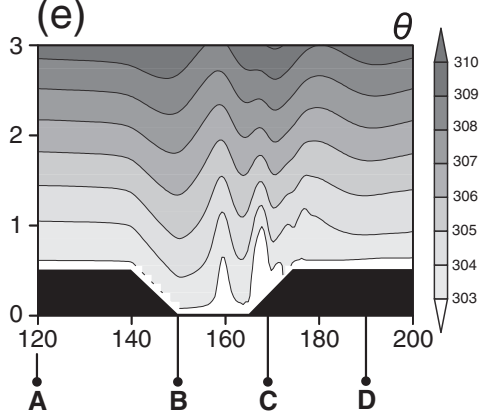

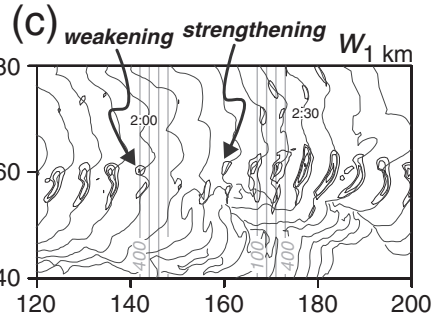

(f)

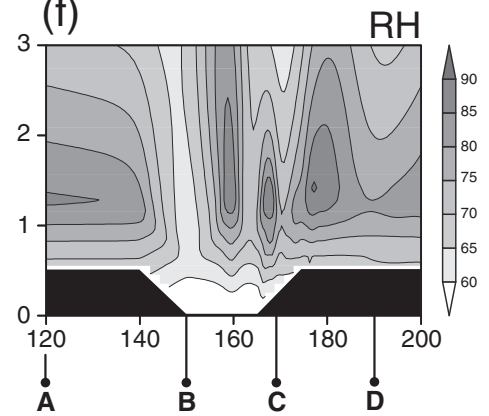

(d)

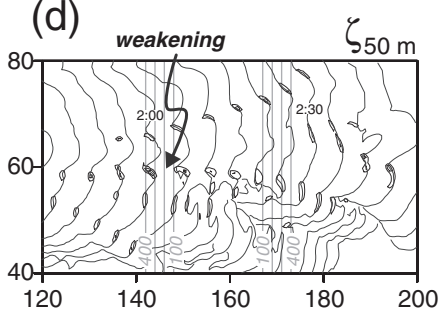

(g)

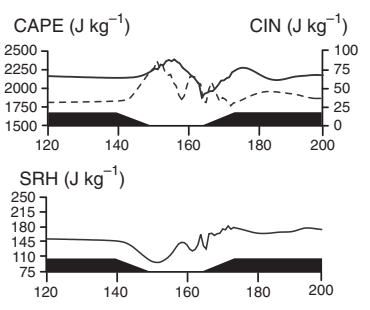

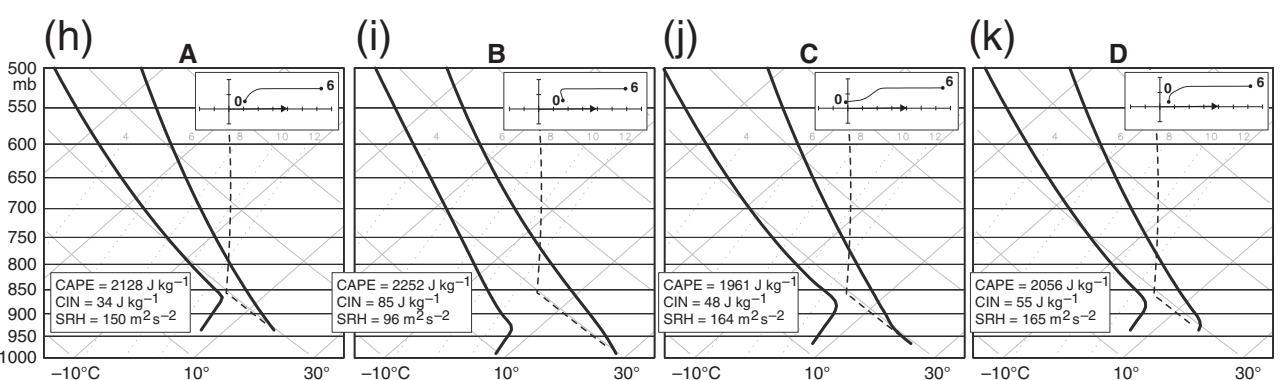

Fig. 12. As in Fig. 10, but for an environmental wind profile having westerly ground-relative winds at the surface far from the valley (Fig. 1d). In (b)-(d), fields are shown every $5 \mathrm{~min}$.

\subsection{0-m tall isolated hill}

We first consider the case of an isolated hill centered at $\left(x_{0}, y_{0}\right)$. The terrain height is given by

$h(x, y)=\frac{h_{0}}{\left[1+\left(\frac{x-x_{0}}{a}\right)^{2}+\left(\frac{y-y_{0}}{a}\right)^{2}\right]^{\frac{3}{2}}}$

where $a$ is roughly the distance from the peak where the terrain height is half the height of the peak. The variables have the following values: $a=10 \mathrm{~km}, h_{0}=500 \mathrm{~m}, x_{0}=$ $145 \mathrm{~km}$, and $y_{0}=50 \mathrm{~km}$.
The terrain is configured so that the same right-moving supercell examined in Sections 3 and 4.1 passes through the cyclonic (positive) vertical vorticity extremum induced by the terrain at approximately $t=2 \mathrm{~h}$ (Fig.13a-e). The most significant cyclonic vorticity anomaly is centered $5 \mathrm{~km}$ southeast of the hilltop and exceeds $7.5 \times 10^{-4} \mathrm{~s}^{-1}$ (Fig.13e). An additional region of enhanced vertical vorticity is found farther downstream ( $\sim 30 \mathrm{~km}$ east-northeast) of the hill, is more transient, and is associated with a region of gravity wave-breaking and turbulence. The most notable change in the evolution of the supercell relative to the control supercell (cf. Figs. $2 \mathrm{i}-\mathrm{l}$ and $13 \mathrm{a}-\mathrm{d}$ ) is gradual strengthening of the midlevel and low-level updraft as the storm encounters a region of upslope winds on the western slope of the hill 
(a)
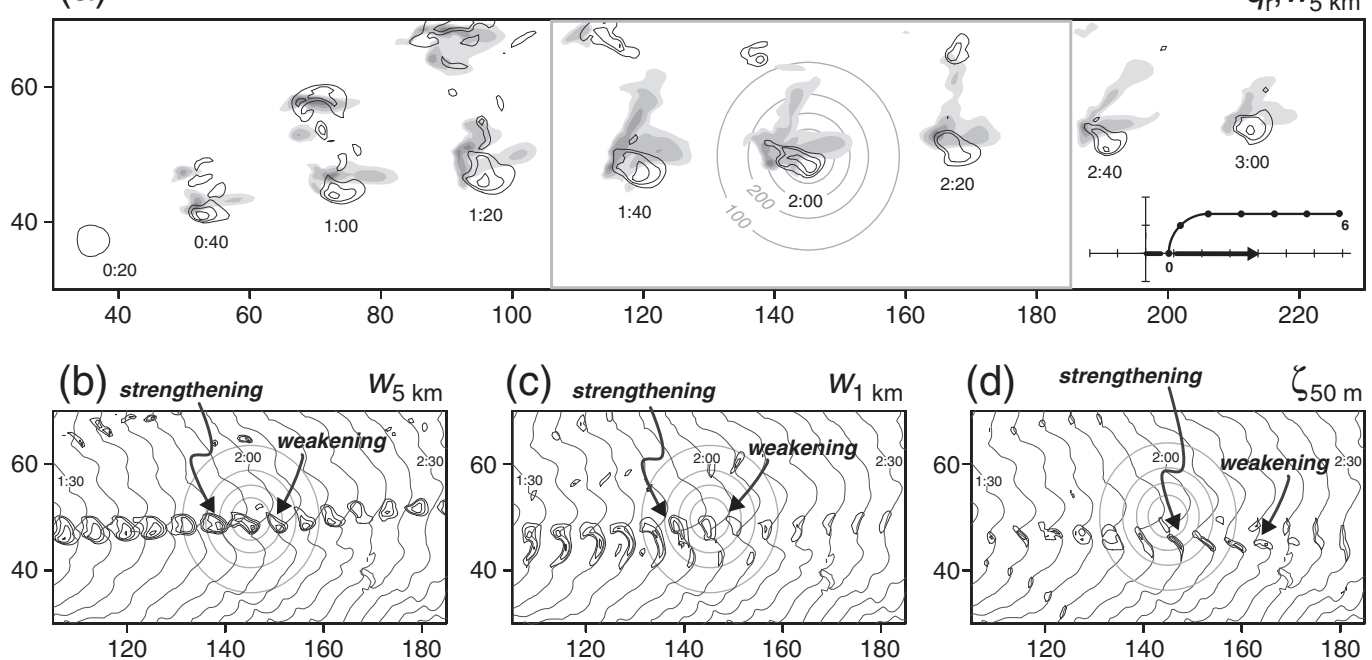

(c) strengthening

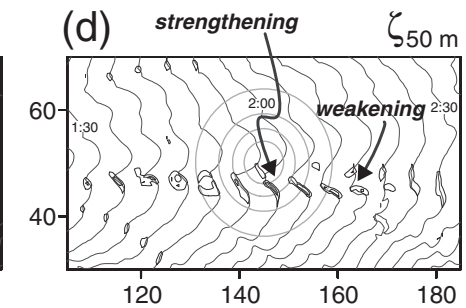

(e)
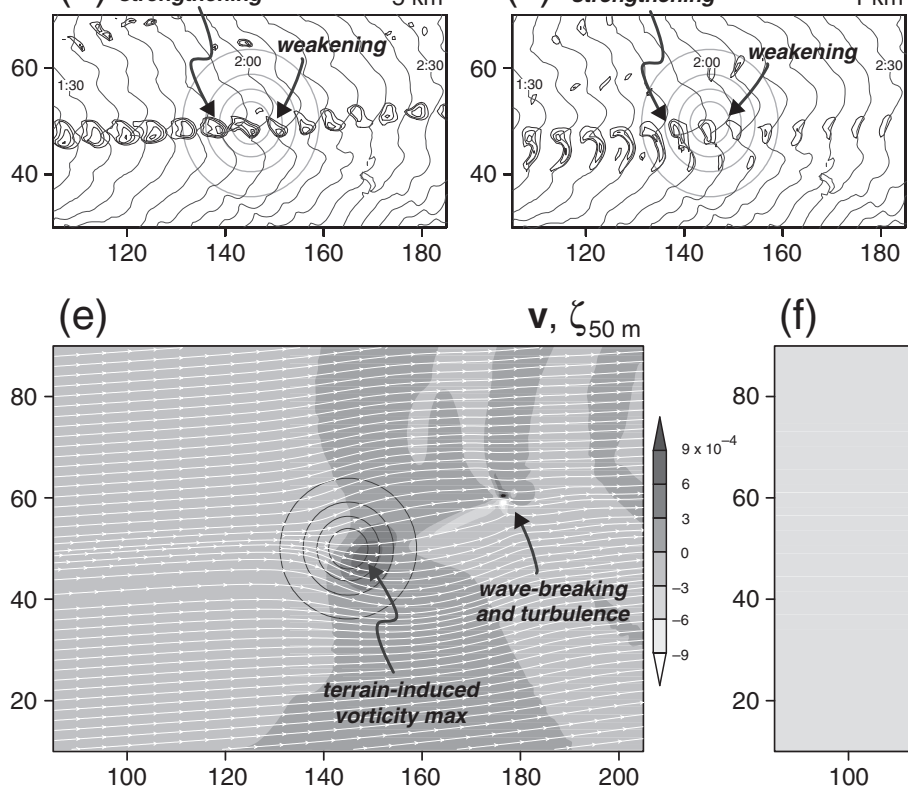

(f)

CAPE

(g)

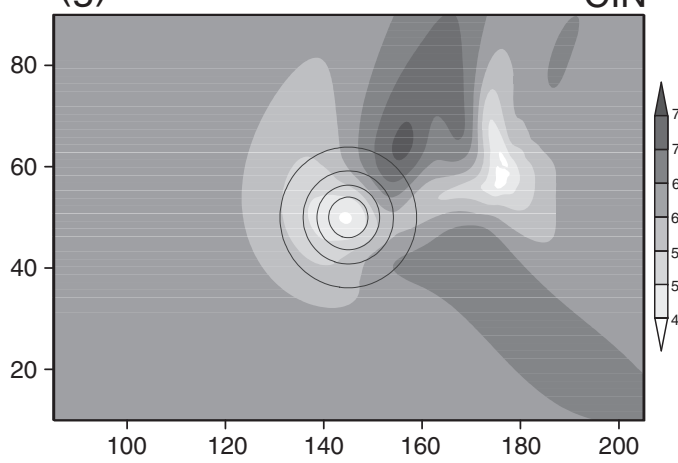

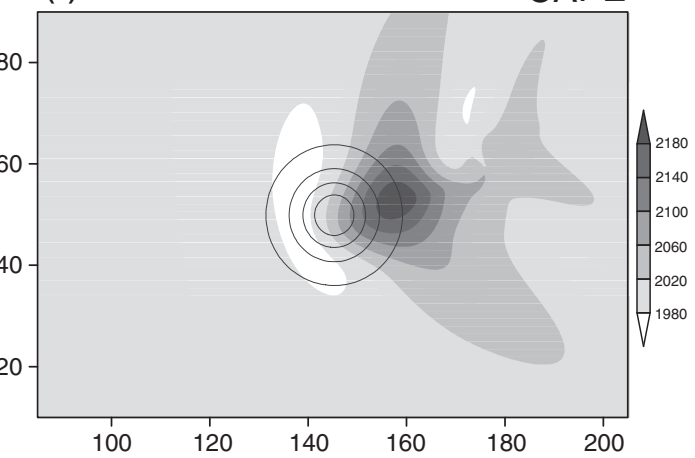

(h)

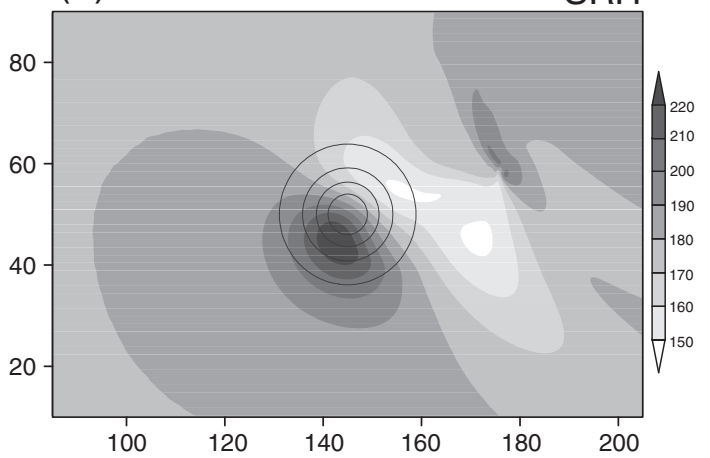

Fig. 13. Model output for simulations for the case of a 500-m tall isolated hill and an environmental wind profile having westerly ground-relative winds at the surface far from the hill (Fig.1d). (a)-(d) As in Figs.14-12. The gray rectangle in (a) encloses the region that is displayed in (b)-(d). (e) Approximately steadystate streamlines (white arrows) at the lowest grid level $(z=50 \mathrm{~m})$ overlaid on the vertical vorticity field ( $\mathrm{s}^{-1}$; shaded) in a simulation with the same terrain configuration but without a storm [the region shown is a bit larger than the region shown in (b)-(d)]. Surface elevations of $100 \mathrm{~m}, 200 \mathrm{~m}$, $300 \mathrm{~m}$, and $400 \mathrm{~m}$ are indicated with black contours. (f) Approximate steady-state CAPE ( $\mathrm{J} \mathrm{kg}^{-1}$ ) in a simulation without a storm. Surface elevations of $100 \mathrm{~m}$, $200 \mathrm{~m}$, $300 \mathrm{~m}$, and $400 \mathrm{~m}$ are indicated with black contours. (g) As in (f), but CIN $\left(\mathrm{J} \mathrm{kg}^{-1}\right)$ is shown. (h) As in (f) and (g), but SRH ( $\left.\mathrm{m}^{2} \mathrm{~s}^{-2}\right)$ is shown.

(Fig. 13b, c), followed by a weakening of the updrafts but a rapid spin-up of low-level vorticity (Fig.13d) as the storm passes through the aforementioned primary cyclonic vorticity anomaly on the lee slope.

The horizontal heterogeneity in the CAPE (Fig.13f), CIN (Fig. 13g), and SRH (Fig. 13h) fields introduced by the hill is complicated, to say the least, especially relative to the heterogeneity observed in the simulations with two-dimensional terrain. The supercell encounters decreasing CIN and increasing SRH on the upslope (west) side of the hill (Fig. 13g, h), which plausibly contributed to the intensification of the updrafts over this stretch. On the other hand, the rapid 
strengthening of low-level rotation in the supercell on the lee slope of the hill seems likely to be the result of the storm encountering the terrain-induced cyclonic vertical vorticity maximum, given that CIN increases and SRH decreases as one moves down the lee slope. Indeed, both the midlevel and lowlevel updrafts weaken as the low-level rotation intensifies. One might imagine that low-level rotation in the storm could respond quickly to an environmental vertical vorticity perturbation, whereas one might expect that the updraft would not be as affected by an environmental vertical vorticity perturbation as much as a change in the thermodynamic conditions and/or vertical wind shear. The low-level rotation in the storm weakens after the storm moves east of the terrain-induced cyclonic vorticity anomaly.

The lee weakening of the supercell is not as severe in this simulation (Fig. 13a-c) as in those with two-dimensional terrain and the same westerly ground-relative wind profile (e.g., Fig. 6a-c). The updraft of the storm in this simulation does not encounter as significant an increase in CIN (nor reduced relative humidity; not shown) in the lee of the hill along its track to the south of the hill (Fig.13g). In an additional simulation (not shown) in which a supercell was initiated farther north, such that it tracked to the north of the hill, the storm weakened noticeably upon encountering the enhanced CIN (and reduced relative humidity) found 10$20 \mathrm{~km}$ northeast of the hilltop (Fig. 13g). This storm also passed through the terrain-induced anticyclonic vertical vorticity anomaly centered roughly $5 \mathrm{~km}$ northwest of the hill (Fig. 13e), but was essentially unaffected by this environmental perturbation, which was much smaller in magnitude than the cyclonic vorticity anomaly located on the southeast flank of the hill (the anomaly does not even result in a closed vertical vorticity contour in Fig. 13e).

\subsection{0- $m$ tall ridge with $10-\mathrm{km}$ wide gap}

Next, we consider the case of a flat-topped ridge with height $h_{0}$, parallel to the $y$ axis, centered at $x_{0}=120 \mathrm{~km}$, with a gap perpendicular to the ridgeline centered at $y_{0}=50 \mathrm{~km}$. This is the same configuration used by Gaberšek and Durran (2004). The terrain height is defined by the product

$h(x, y)=r(x, y) g(y)$.

The shape of the ridge into which the gap is incised is given by

$r(x, y)= \begin{cases}\frac{h_{0}}{16}\left[1+\cos \left(\frac{\pi s}{4 a}\right)\right]^{4}, & s \leq 4 a \\ 0, & \text { otherwise }\end{cases}$

where

$s= \begin{cases}\max \left(0,\left|x-x_{0}\right|-b\right), & \left|y-y_{0}\right| \leq c \\ \max \left\{0,\left[\left(x-x_{0}\right)^{2}+\left(\left|y-y_{0}\right|-c\right)^{2}\right]^{\frac{1}{2}}-b\right\}, & \text { otherwise }\end{cases}$

The width of the top of the flat-topped ridge is $2 b$ in the $x$ direction and $2(b+c)$ in the $y$ direction. The ends of the flattopped ridge are semicircular with radius $b$. The slopes of the ridge have an approximate half-width $a$. The gap is carved out of the ridge by multiplying $r(x, y)$ by

$$
g(y)= \begin{cases}0, & \left|y-y_{0}\right| \leq \frac{d}{2} \\ \sin \left[\frac{\pi\left(\left|y-y_{0}\right|-\frac{d}{2}\right)}{2 e}\right], & \frac{d}{2}<\left|y-y_{0}\right|<e+\frac{d}{2} \\ 1, & \text { otherwise }\end{cases}
$$

where $d$ is the width of the floor of the gap and $e$ is the horizontal distance over which the sidewalls rise from the floor of the gap to the ridgeline. The values of $a, b, d$, and $e$ are $10 \mathrm{~km}$, and the value of $c$ is $100 \mathrm{~km}$.

As was case for the isolated hill introduced in Section 5.1, the terrain described above is configured so that the rightmoving supercell passes through the cyclonic (positive) vertical vorticity extremum induced by the terrain at approximately $t=2 \mathrm{~h}$ (Fig. 14a-e). As was also the case in the simulation presented in Section 5.1, the upstream wind profile strongly favors the cyclonic lee vorticity maximum (located northeast of the gap; Fig. 14e) over the anticyclonic vorticity maximum (located southeast of the gap; Fig.14e), for reasons beyond the scope of this presentation. The primary cyclonic vorticity maximum has a magnitude of approximately $7 \times 10^{-4} \mathrm{~s}^{-1}$ (Fig.14e). Additional vertical vorticity perturbations are induced by the interaction of the environmental winds and gap/ridge, particularly east-southeast of the gap, but these are smaller in horizontal scale and transient, albeit occasionally stronger in magnitude (occasionally $>10^{-3} \mathrm{~s}^{-1}$ ) than the primary cyclonic vorticity anomaly northeast of the gap (Fig. 14e). As was the case in the simulation with an isolated hill, these transient, smallerscale vorticity perturbations are in regions of gravity wave breaking and turbulence. In addition to the terrain-induced heterogeneity in the horizontal wind field, the terrain is responsible for horizontal heterogeneity in the CAPE (Fig.14f), CIN (Fig.14g), and SRH (Fig.14h) fields that is even more complex than the heterogeneity seen in the isolated hill simulations of Section 5.1.

As the supercell nears the ridge that is incised by the gap, the storm encounters enhanced SRH on the windward slope of the ridge, north of the gap in the ridge (Fig. 14h). Similar to the case of a two-dimensional hill (and same environmental wind profile in the far field; Fig. 1d), the windward slope also is a region of reduced CIN (Fig. 14g) and enhanced relative humidity (not shown). Not surprisingly, the supercell undergoes some modest strengthening in terms of its updraft and vertical vorticity (Fig. 14b-d) as it approaches the longitude of the ridge, before it reaches the terrain-induced cyclonic vertical vorticity anomaly. Over the ensuing $20 \mathrm{~min}$, the storm passes over the lee slope and the terrain-induced cyclonic vorticity maximum (Fig. 14b-e), but also encounters increasing CIN (Fig.14g), reduced relative humidity (not shown), and decreasing SRH (Fig.14h). The net result is a weakening of the updraft (Fig. 14b, c), but the supercell's lowlevel mesocyclone maintains its intensity (the maximum vertical vorticity at the lowest grid level actually increases slightly) (Fig.14d). The low-level mesocyclone eventually 
(a)

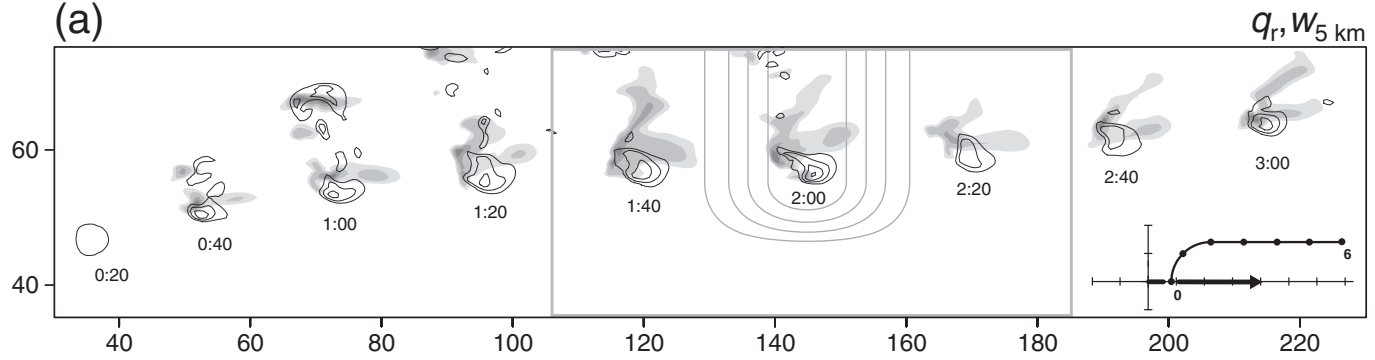

(b)

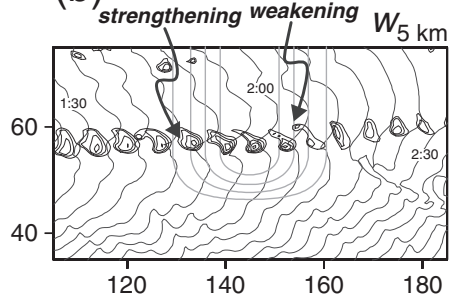

(c)

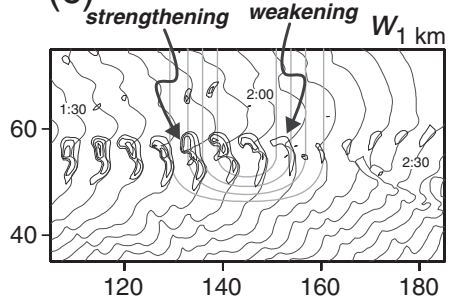

(d)
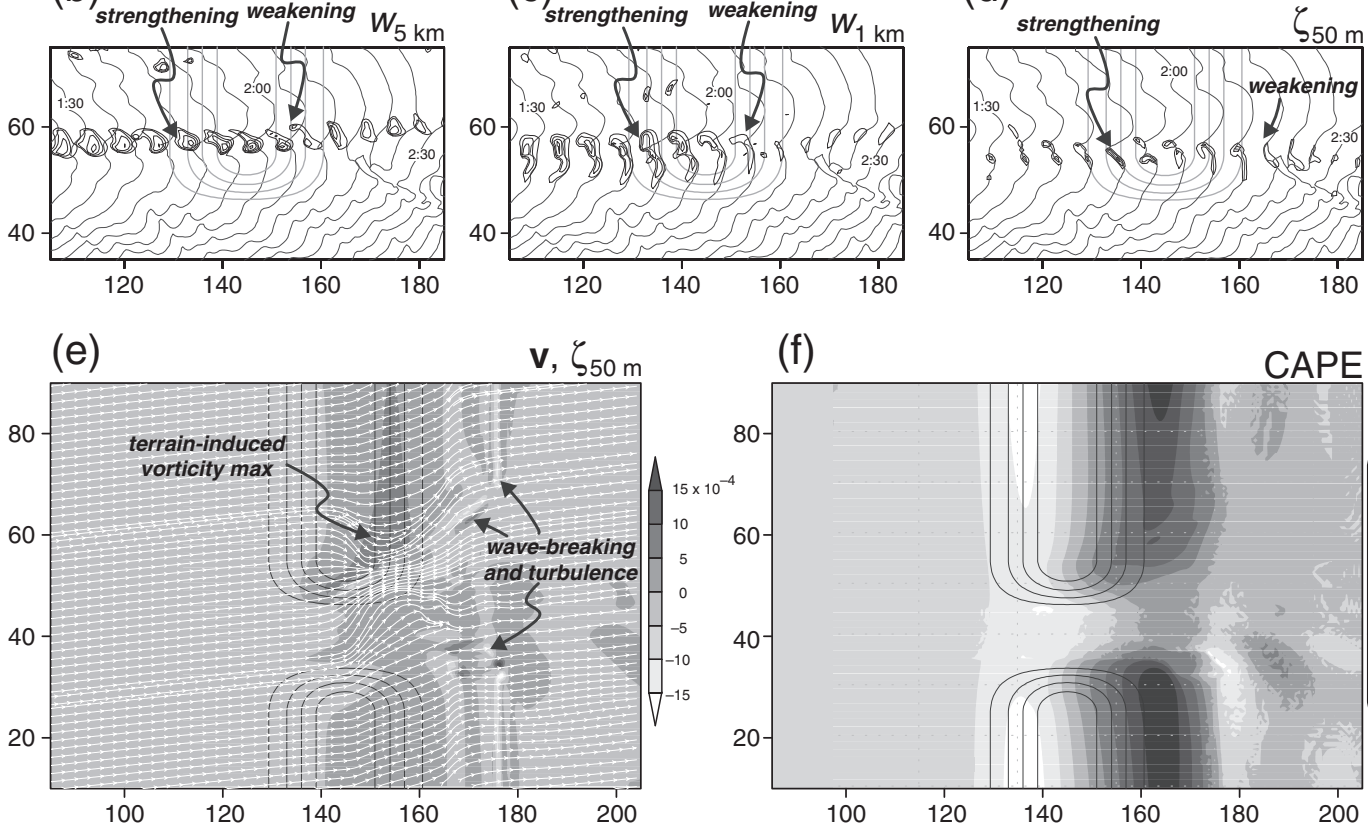

(f)

(g)

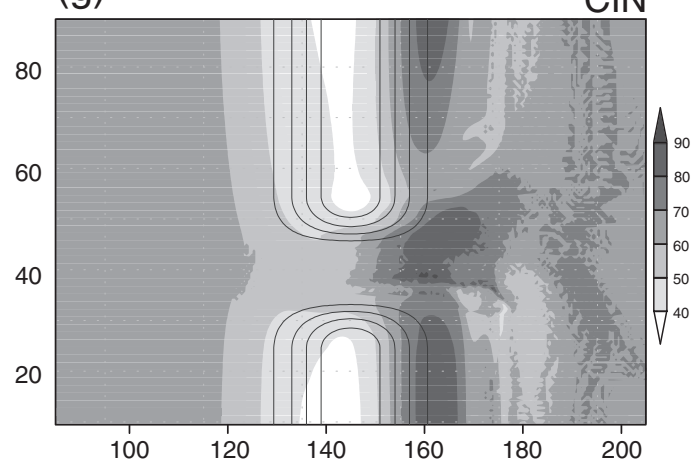

CAPE

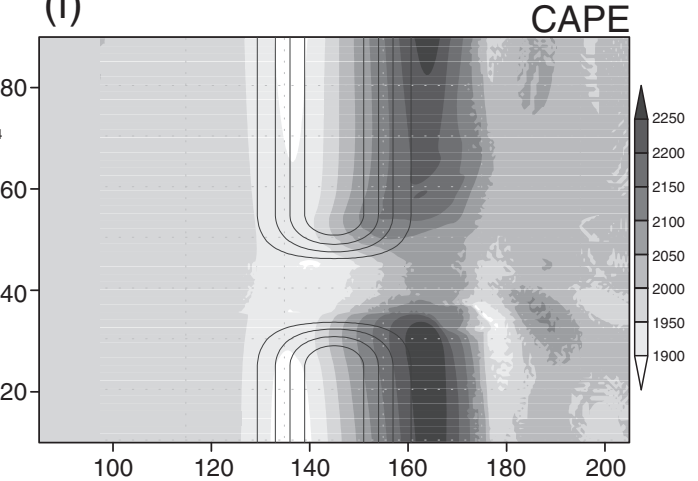

(h)

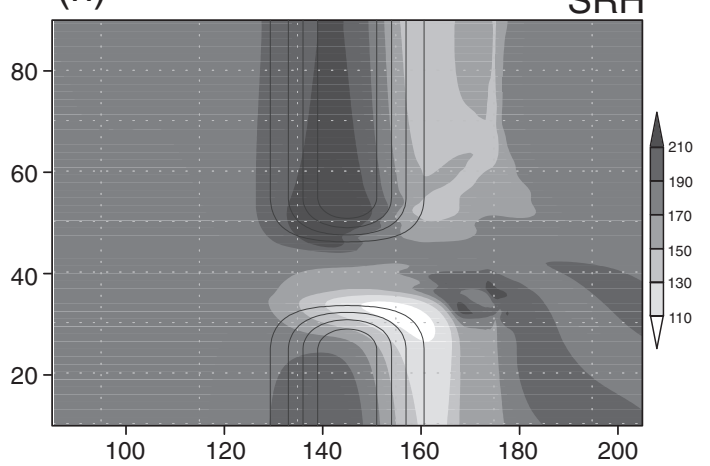

Fig. 14. As in Fig. 13, but for the case of a 10-km wide gap incised in 500-m tall ridge.

weakens when the supercell moves east of the pre-existing terrain-induced vertical vorticity maximum. The motion of the supercell is slightly to the left of its initial motion during its weak phase in the lee of the ridge. The storm updraft and mesocyclone eventually regain their far upstream intensities (the eastward motion also resumes) once the storm has moved far east of the ridge by $t=3 \mathrm{~h}$ (Fig. 14a).
In summary, the supercell was affected by the terrain in a similar manner as in the case of the two-dimensional ridge (Section 4.1; Fig.6a-d), with enhancement of the storm occurring on the windward slope and weakening occurring on the lee slope, and the trends being correlated with changes in CIN and SRH (and relative humidity), as discussed in prior sections. In the fortuitous case of a supercell passing over a 
terrain-induced vertical vorticity anomaly that may result from three-dimensional variations in the terrain height, the low-level mesocyclone may be able to maintain strength or even intensify despite the generally negative influence of the increased CIN and decreased SRH on the updraft. In additional simulations (not shown) in which the storm crossed the ridge at different latitudes such that the storm did not pass over a mesoscale vorticity anomaly induced by the terrain, the evolution of the storm mirrored the evolution in simulations without a gap; that is, the updraft weakening in the lee of the ridge was not generally accompanied by strengthening of the low-level mesocyclone.

\section{Summary and closing remarks}

This article has presented numerical simulations designed to study the effects of idealized terrain on a right-moving, cyclonically rotating supercell. To a large extent, in simulations with two-dimensional terrain, changes in storm intensity (relative to the storms in flat-terrain control simulations) could be attributed to changes in the environment that are associated with airflow over the terrain, with the environment on the lee slope of a hill or escarpment, or where terrain drops into a valley, being more hostile to the storms in terms of CIN and relative humidity than the far-field environment and windward-slope environment, or where terrain rises out of a valley (the terrain also led to heterogeneity in the vertical windshear fields but this did not seem to be as important as the effects on the thermodynamic fields). In principle, storm dynamics only depend on the storm-relative wind profile (neglecting the effects of surface friction), and therefore only on the length and curvature of the hodograph trace. However, the horizontal heterogeneity induced by the interaction of the environmental winds and terrain that was found to be so important in this study is vitally dependent on the groundrelative wind profile, and therefore on the location of the hodograph trace relative to the origin.

Regions where isentropic surfaces were depressed relative to their far-field heights were generally regions of enhanced $\mathrm{CIN}$, reduced relative humidity, and storm weakening, in terms of updraft and mesocyclone strength. The flow over the idealized terrain features excites gravity waves, and the details of the resultant wind and thermodynamic fields are more complicated than in most theoretical and numerical studies of terrain-forced gravity waves, as these tend to use much simpler vertical wind and static stability profiles than the profiles used herein. For example, in theoretical and numerical studies of terrain-forced gravity waves, the upstream environment is often defined to have a static stability and horizontal wind that are constant with height (e.g., Long, 1953; Lilly and Klemp, 1979), and when vertical variation is introduced, it often is introduced by way of specifying a simple two-layer upstream environment (e.g., Durran, 1986). In contrast, though our idealized environmental wind and thermodynamic profiles are relatively simple compared to what is often observed in actual supercell environments, our profiles have vertically varying static stability, vertically varying wind shear, and vertical variations in the terrain-relative wind direction (e.g., the profile in Fig. 1b has low-level easterlies and upper-level westerlies). Predicting the details of how even fairly simple terrain will influence the airflow above is itself a very difficult problem outside of a limited number of idealized situations (many have devoted a significant fraction of their careers to studying this problem alone!). If one can ascertain how the terrain will affect the isentropic surfaces, then it seems fairly straightforward to determine the effects of the terrain on environmental CIN and relative humidity, and ultimately the effects of the terrain on the overlying storm.

When three-dimensional terrain is introduced, the effect of terrain on the storm environment can be further complicated by the generation of mesoscale vorticity anomalies. It generally might be difficult to predict the structure of any lee vortices that might develop (and might subsequently be encountered by storms), given the complexity of the upstream soundings and wind profiles that often accompany severe storm environments (e.g., hodograph curvature and inflection points in wind profiles). Furthermore, even if one could predict the impact of terrain on the downstream horizontal wind field, we suspect that, in practice, it would be hard to anticipate whether any lee vorticity anomalies might be able to influence low-level rotation in the storm given that other factors in the lee generally lead to unfavorable sounding and hodograph modifications. Our overall sense is that horizontal heterogeneity in the $\mathrm{CIN}, \mathrm{SRH}$, and relative humidity fields introduced by three-dimensional terrain influences storms in more important ways than terraininduced vertical vorticity extrema that a storm might fortuitously pass over.

In the present study we only have considered supercells. We believe that one important difference between this investigation and an investigation of how cold-pool-driven convection (e.g., a squall line) is modified by terrain (e.g., Frame and Markowski, 2006) is that in the case of cold-pooldriven convection, one might be more concerned with how outflow behaves going over a ridge, escarpment, etc. (e.g., whether or not a hydraulic jumps formed in outflow), given that such convection is maintained by cells being repeatedly triggered by the gust front. Supercells are not as reliant on gust front lifting, however. Rather, supercells are primarily maintained by lifting forced by dynamic vertical pressure gradient forces that act over a much deeper layer than the depth of the outflow (e.g., Rotunno and Klemp, 1982). In the supercell simulations conducted herein, it appears that the influence of the terrain on the environmental air is what is most relevant.

It ought to be apparent to the reader that this study has raised a lot more questions that it has answered. The present article might be viewed best as a pilot study. It is our feeling that we have only scratched the surface. For example, though the environmental heterogeneity generated by the airflow over terrain could explain much of the behavior of the simulated storms, we certainly are unable to explain everything in terms of perturbations in environmental CAPE, CIN, SRH, etc. This really should not be surprising-after all, storms are unsteady even in horizontally homogeneous environments. Moreover, our focus was on the attributes of the updraft and near-surface mesocyclone; however, the vertical velocity at $z=1 \mathrm{~km}$ and $z=5 \mathrm{~km}$, and the vertical vorticity at $z=50 \mathrm{~m}$ are not the only important aspects of storms. On the contrary, there are virtually innumerable storm traits that we have not investigated in this study, for 
example, the changes in the microphysical characteristics of the storm, depth of the outflow, gust front speed, precipitation, etc. We only reported on a few in this study. Yet some of these other aspects of the simulated storms could be important as well. For example, the depth of the outflow plays a major role in the maintenance of the cold-pool-driven convective storms mentioned above, and even though dynamic vertical pressure gradients acting over a large fraction of the storm depth are a crucial aspect of supercell sustenance, supercell structure and evolution are not entirely independent of what goes on along the gust front (e.g., Ziegler et al., 2010). A storm that develops over relatively high terrain would tend not to possess outflow as cold and deep as a storm that develops over relatively low terrain because of the lower cloud base height in the former environment (cf.Fig. 8h and $\mathrm{k}$ ). If the storm suddenly encounters lower terrain by passing over an escarpment or into a valley, the storm might weaken regardless of the ground-relative wind direction (and be weaker than a storm that spends its entire life over the lower terrain) until the storm's outflow can have time to deepen over the lower terrain. Such behavior was observed in the simulations described in Sections 4.2 and 4.3 (e.g., at least temporary weakening was observed when storms crossed from high flat terrain to low flat terrain regardless of the wind profile).

Some additional paths for future work might be to expand the parameter space to different low-level stratifications, terrain amplitudes, and ground-relative wind speeds. One might naively assume that the effects of terrain on the environment, and ultimately on the storm, would decrease as the boundary layer stratification, terrain amplitude, and ground-relative winds decrease. On the other hand, slow ground-relative winds would tend to yield slow storm motions, and a slow-moving storm might have a longer residence time within a region where the environment is perturbed, albeit not perturbed as greatly as in a situation with faster winds blowing over the terrain (i.e., there might well be offsetting effects that need to be explored further). There are obviously many more terrain configurations that can be explored as well, and even two-dimensional terrain can produce complicated outcomes (e.g., a simple valley like that in Section 4.3 affords the opportunity for wave interactions). Lastly, terrain can produce environmental heterogeneity in ways that the present suite of simulations was not designed to replicate. For example, sometimes the channeling of air by terrain (e.g., the airflow along a valley; Dotzek, 1999, 2001) can lead to the superpositioning of air masses having different source regions, which we suspect could produce variations in CAPE and CIN that are more important than the CAPE and CIN perturbations associated with terrain-generated gravity waves. We believe that there is still much to be explored and that the problem might be well-suited for partnerships between researchers and forecasters.

\section{Acknowledgments}

The lead author is grateful for the support of the Deutsches Zentrum für Luft- und Raumfahrt (DLR), Institut für Physik der Atmosphäre, where he was a Visiting Scientist in the summer and fall of 2009. We thank George Bryan for allowing us to use his numerical model. A large fraction of the figures were created using the Grid Analysis and Display System (GrADS), developed by the Center for Ocean-Land-Atmosphere Studies.

\section{References}

Bosart, L.F., Seimon, A., LaPenta, K.D., Dickinson, M.J., 2006. Tornadogenesis over complex terrain: the Great Barrington, Massachusetts, Tornado on 29 May 1995. Wea. Forecasting 21, 897-922.

Bryan, G.H., 2002. An investigation of the convective region of numerically simulated squall lines. Ph.D. thesis, The Pennsylvania State University, $181 \mathrm{pp}$.

Bryan, G.H., Fritsch, J.M., 2000. Moist absolute instability: the sixth static stability state. Bull. Amer. Met. Soc. 81, 1207-1230.

Bryan, G.H., Fritsch, J.M., 2002. A benchmark simulation for moist nonhydrostatic numerical models. Mon. Wea. Rev. 130, 2917-2928.

Ćurić, M., Janc, D., Vučković, V., 2007. Numerical simulation of a Cb cloud vorticity. Atmos. Res. 83, 427-434.

Deardorff, J.W., 1980. Cloud top entrainment instability. J. Atmos. Sci. 37, 131-147.

Dotzek, N., 1999. Mesoskalige numerische Simulation von Wolken- und Niederschlagsprozessen über strukturiertem Gelände (Mesoscale numerical simulation of cloud and precipitation processes over structured/ complex terrain). Dissertation, Institut für Meteorologie und Klimaforschung, Universität Karlsruhe (TH), 127 pp. [In German. Available at www.essl.org/people/dotzek/].

Dotzek, N., 2001. Tornadoes in Germany. Atmos. Res. 56, 233-251.

Durran, D.R., 1986. Another look at downslope windstorms. Part I: the development of analogs to supercritical flow in an infinitely deep, continuously stratified fluid. J. Atmos. Sci. 43, 2527-2543.

Durran, D.R., Klemp, J.B., 1983. A compressible model for the simulation of moist mountain waves. Mon. Wea. Rev. 111, 2341-2361.

Epifanio, C.C., Durran, D.R., 2002. Lee-vortex formation in free-slip stratified flow over ridges. Part I: comparison of weakly nonlinear inviscid theory and fully nonlinear viscous simulations. J. Atmos. Sci. 59, 1153-1165.

Frame, J.W., Markowski, P.M., 2006. The interaction of simulated squall lines with idealized mountain ridges. Mon. Wea. Rev. 134, 1919-1941.

Frame, J.W., Markowski, P.M., 2010. Numerical simulations of radiative cooling beneath the anvils of supercell thunderstorms. Mon. Wea. Rev. 138, 3024-3047.

Frame, J.W., Markowski, P.M., Petters, J.L., 2008. The dynamical influences of cloud shading on simulated supercell thunderstorms. Preprints 24th Conf. on Severe Local Storms. Amer. Meteor. Soc, Savannah, GA.

Frame, J.W., Petters, J.L., Markowski, P.M., Harrington, J., 2009. An application of the tilted independent pixel approximation to cumulonimbus environments. Atmos. Res. 91, 127-136.

Gaberšek, S., Durran, D.R., 2004. Gap flows through idealized topography. Part I: forcing by large-scale winds in the nonrotating limit. J. Atmos. Sci. 61, 2846-2862.

Gal-Chen, T., Somerville, R., 1975. On the use of a coordinate transformation for the solution of the Navier-Stokes equations. J. Comput. Phys. 17, 209-228.

Hannesen, R., Dotzek, N., Gysi, H., Beheng, K.D., 1998. Case study of a tornado in the Upper Rhine valley. Meteorol. Z. 7, 163-170.

Hannesen, R., Dotzek, N., Handwerker, J., 2000. Radar analysis of a tornado over hilly terrain on 23 July 1996. Phys. Chem. Earth B 25, 1079-1084.

Knopfmeier, K.H., Markowski, P.M., Richardson, Y.P., 2008. Numerical simulations of supercells in convective boundary layers. Preprints 24th Conf. on Severe Local Storms. Amer. Meteor. Soc, Savannah, GA.

LaPenta, K.D., Bosart, L.F., Galarneau Jr., T.J., Dickinson, M.J., 2005. A multiscale examination of the 31 May 1998 Mechanicville, New York, tornado. Wea. Forecasting 20, 494-516.

Lilly, D.K., Klemp, J.B., 1979. The effects of terrain shape on nonlinear hydrostatic mountain waves. J. Fluid Mech. 95, 241-261.

Lin, Y.-L., Farley, R.D., Orville, H.D., 1983. Bulk parameterization of the snow field in a cloud model. J. Climate Appl. Meteor 22, 1065-1092.

Long, R.R., 1953. Some aspects of the flow of stratified fluids I. A theoretical investigation. Tellus 5, 42-58.

Markowski, P.M., Harrington, J., 2005. A simulation of a supercell thunderstorm with emulated radiative cooling beneath the anvil. J. Atmos. Sci. 62, 2607-2617.

Nowotarski, C., Markowski, P., Richardson, Y., Bryan, G., 2010. Simulating supercell thunderstorms in a horizontally heterogeneous convective boundary layer. Preprints, 25th Conference on Severe Local Storms, Denver, Colorado, Amer. Meteor. Soc. 
Reeves, H.D., Lin, Y.-L., 2007. The effects of a mountain on the propagation of a preexisting convective system for blocked and unblocked flow regimes. J. Atmos. Sci. 64, 2401-2421.

Rotunno, R., Klemp, J.B., 1982. The influence of the shear-induced pressure gradient on thunderstorm motion. Mon. Wea. Rev. 110, 136-151.

Smolarkiewicz, P.K., Rotunno, R., 1989. Low Froude number flow past threedimensional obstacles. Part I: baroclinically generated lee vortices. J. Atmos. Sci. 46, 1154-1166.
Weisman, M.L., Klemp, J.B., 1982. The dependence of numerically simulated convective storms on vertical wind shear and buoyancy. Mon. Wea. Rev. $110,504-520$.

Wicker, L.J., Skamarock, W.C., 2002. Time splitting methods for elastic models using forward time schemes. Mon. Wea. Rev. 130, 2088-2097.

Ziegler, C.L., Mansell, E.R., Straka, J.M., MacGorman, D.R., Burgess, D.W., 2010. The impact of spatial variations of low-level stability on the life cycle of a simulated supercell storm. Mon. Wea. Rev. 138, 1738-1766. 